\title{
GPS-Tracking in touristischen Destinationen - Neue Software- Anwendung zur Erfassung des Mobilitätsverhaltens am Beispiel von Wanderern im Harz
}

\author{
Sven Groß ${ }^{1} \cdot$ Anne Menzel $^{2}$
}

Eingegangen: 26. März 2015 / Angenommen: 12. Januar 2016 / Online publiziert: 4. Februar 2016

(C) Springer-Verlag Berlin Heidelberg 2016

Zusammenfassung Zur Analyse des Mobilitätsverhaltens von Personen gibt es eine Vielzahl quantitativer und qualitativer Methoden. GPS-Tracking hat sich in den letzten Jahren zu einem festen Bestandteil bei der Erforschung des (touristischen) Mobilitätsverhaltens entwickelt. Basierend auf den - beispielsweise durch GPSDatenlogger - erfassten Strecken lässt sich das raum-zeitliche Verhalten von Personen analysieren. Weitergehende Fragestellungen, z. B. zu deren Handlungen im Sinne von Aktivitäten am „Point of Interest“ (PoI), zur Motivation (beispielsweise für das Verweilen an einem bestimmten PoI), zu personenbezogenen Daten oder zur Zufriedenheit können durch eine Kombination mit anderen Erhebungstechniken erfasst werden. Eine eigens an der Hochschule Harz entwickelte Android-Applikation für Tablet-Computer ermöglicht die Verknüpfung sich ergänzender empirischer Techniken: GPS-Tracking und Face-to-Face-Befragung. Die Besonderheit liegt hierbei in der entwickelten Software, dem GimToP-Toolkit (GTK). Mit dessen Hilfe ist es möglich, direkt im Anschluss an die zurückgelegte Strecke die Tracks inklusive Pausen in einer interaktiven Karte darzustellen. Somit können die Probanden direkt nach Zurücklegen ihrer Strecke zu den einzelnen Wegepunkten, an denen sie länger verweilten, befragt werden. Dies stellt einen neuen, aussichtsreichen Ansatz für die Erforschung des raum-zeitlichen Verhaltens von Personen (z. B.

Prof. Dr. Sven Groß

sgross@hs-harz.de

1 Fachbereich Wirtschaftswissenschaften, Institut für Tourismusforschung, Hochschule Harz, Friedrichstr. 57-59, 38855 Wernigerode, Deutschland

2 Stabstelle Forschung, Hochschule Harz, Friedrichstr. 57-59, Wernigerode, Deutschland
Wanderern, Städtetouristen, Besuchern von Freizeiteinrichtungen und Sehenswürdigkeiten) dar. Die wichtigsten Ziele, Elemente und Möglichkeiten der neuen Software werden im vorliegenden Beitrag ebenso dargestellt wie Ergebnisse einer Beispieluntersuchung mittels des GTK von Wanderern am Brocken.

Schlüsselwörter Mobilitätsanalyse · GPS-Tracking · Touristisches Mobilitätsverhalten · Android-Applikation · Geoinformationssystem

\section{GPS Tracking at Tourist Destinations-A New Software for the Analysis of Tourist Mobility Behavior Illustrated by Hikers in the Harz Mountains}

\begin{abstract}
There is a variety of quantitative and qualitative methods available to analyze the mobility behavior of people, and GPS tracking has evolved into a major tool to investigate tourism mobility behavior. For example, the use of GPS data loggers enables the recording and analysis of routes and time-space movements of participants. Through the combination of research tools, additional data can be collected, such as activities at the Point of Interest (PoI), motivations (e.g., for spending time at a specific PoI), demographic data and visitor satisfaction. An Android Application for tablets developed by the Harz University allows the link between complementary data-collection tools: GPS tracking and face-to-face surveys. The software GimToP (GTK) enables the researcher to collect data in-situ, and subsequently displays specific results (e.g., track, distance, breaks) in an interactive map. This way, respondents can be asked to provide data during their experience at various track points, which represents a new
\end{abstract}


and significant tool to understand the mobility and spacetime behavior of a variety of respondent groups, such as hikers, city tourists, as well as visitors of leisure facilities or tourist attractions. This presentation will introduce the most important goals and opportunities of this new software, underpinned by a case study presenting the used GTK for hikers in the Harz Mountains.

Keywords Mobility analysis · GPS tracking · Tourist mobility behavior - Android application . Geographic information systems

\section{Einleitung}

Ein möglichst umfassendes Bild vom raum-zeitlichen Verhalten von Personen zu erhalten, ist seit mehreren Jahrzehnten Gegenstand verschiedener wissenschaftlicher Disziplinen bzw. deren Teildisziplinen wie der Geographie, Sozial-, Tourismus- und Verkehrswissenschaften. Hierbei gibt es vielfältige quantitative und qualitative Methoden für die Erforschung der Mobilität (in touristischen Destinationen ${ }^{1}$ ).

Der Begriff Mobilität geht auf „mobilitas“ zurück, was nicht nur Beweglichkeit, sondern auch Schnelligkeit, Gewandtheit, Unbeständigkeit, Wankelmut und Biegsamkeit bedeutet und im Allgemeinen die Beweglichkeit von Personen und Sachen rein physischer Art und bei Personen zusätzlich auch die Beweglichkeit in geistiger oder sozialer Hinsicht mit umfasst. Mobilität kann aber nicht nur als realisierte Beweglichkeit, sondern auch als Fähigkeit zur Beweglichkeit verstanden werden, unabhängig davon, ob von dieser Fähigkeit Gebrauch gemacht wird (vgl. Kleehaupt 1997, S. 9; Canzler/Knie 1998, S. 30; Diewitz/ Klippel/Verron 1998, S. 72; Zängler 2000, S. 19).

Um den Mobilitätsbegriff weiter zu differenzieren, werden oft drei Kategorien herangezogen (vgl. Abb. 1). Dies sind die soziale, informationale und räumliche (physische) Mobilität (vgl. Cerwenka 1999, S. 35; Zängler 2000, S. 20). Andere Autoren sprechen von fünf ineinandergreifenden Mobilitätskategorien, so etwa beim Ansatz des „new mobilities paradigm“, wie er erstmals von Sheller/ Urry 2006 propagiert wurde. Die fünf „,mobilities“, Mobilitäts-Aspekte des „new mobilities paradigm“ sind: a) „physical travel“, d. h. die tatsächliche Ortsveränderung von Personen zum Zwecke der Arbeit, zum Einkaufen, beim Umzug usw., b) ,physical movement“, der Transport von Gütern, c) „imaginative travel“, Reisen im Raum als der durch Filme, Bücher, Erinnerungen, Träume u. Ä. geprägten

\footnotetext{
${ }^{1}$ Unter einer Destination wird ein geographischer Raum (z. B. ein großes Hotel, Resort, ein Ort, eine Region, ein Land) verstanden, den ein Gast als Reiseziel wählt (vgl. Bieger 2008, S. 57).
}

„kognitiven Landkarte“, d) „virtual travel“ (Surfen im Internet) und e) „communicative travel“ durch persönliche Kontakte in Netzwerken über Briefe, Postkarten, Telefon, SMS, E-Mails, Skype, Videokonferenz usw. (vgl. Büscher/ Urry 2009, S. 101 f.). Die in Abb. 1 dargestellte Auslegung von Mobilität geht über diese „mobilities“ noch hinaus, weil auch die soziale (vgl. Franz 1984) sowie die lebensstilspezifische Mobilität (,lifestyle mobility“) (vgl. Cohen/Duncan/ Thulemark 2015) einbezogen werden. ${ }^{2}$

In der vorliegenden Untersuchung ist hauptsächlich die (Verkehrs-) Mobilität ${ }^{3}$ von Interesse, wobei unter Mobilität die Ortsveränderung von Personen verstanden wird, die von einem bestimmten Standort ausgeht (vgl. Kleehaupt 1997, S. 15; Diewitz/Klippel/Verron 1998, S. 72; Zängler 2000, S. 21). Die weiteren Felder der sozialen, informationalen und räumlichen Mobilität (Lifestyle- und Wanderungsmobilität) werden ausgeklammert, da sich diesbezügliche Mobilitätsprobleme, -ansprüche und -verhaltensweisen grundsätzlich von jenen der Personenmobilität unterscheiden.

Untersuchungen zur Mobilität schenken bisher den Vor-Ort-Entscheidungen (auch „in-situ decisions“ bzw. „in-situ evaluation“) und Tätigkeiten an den einzelnen

${ }^{2}$ Die soziale Mobilität beschreibt hierbei zum einen die vertikale Mobilität zwischen gesellschaftlichen Schichten und die horizontale Mobilität zwischen den gesellschaftlichen Gruppen innerhalb einer Schicht. Soziale Mobilität bezeichnet generell Positionsveränderungen von Personen in einem sozialen System (vgl. Franz 1984, S. 23 ff.). Informationale Mobilität kann weiter in geistige Mobilität eines Individuums und mediengebundenen Austausch von Informationen zwischen Personen unterteilt werden. Letzteres kann sowohl beispielsweise die Sprache als imaginäre Mobilität an einen anderen Ort oder die virtuelle Mobilität im Internet (z. B. Videokonferenz oder „Second Life“) betreffen. Die räumliche Mobilität meint ,(..) die Veränderung mobilitätsfähiger Einheiten zwischen zwei Elementen im geographischen Raum“ (Kaiser 1993, S. 29). Mit Wanderungsmobilität, als Teil der räumlichen Mobilität, werden die räumlichen Bewegungen von Personen zusammengefasst, mit denen ein dauerhafter Wechsel der Wohnung oder des Wohnortes verbunden ist (vgl. Hautzinger/Pfeiffer/Tassaux-Becker 1994, S. 12). Wanderungsmobilität meint eine einseitige Raumüberwindung von A nach B ohne zurückzukehren, wohingegen (Verkehrs-) Mobilität die Rückkehr von B nach A bedeutet, wenn die in B zu tätigende Aktivität erledigt ist. Lifestyle Mobilität wird als wiederkehrender, unterschiedlich langer, aber doch zeitweiliger Wechsel des gewöhnlichen Aufenthaltsortes definiert. Mit Lifestyle-Mobilität werden mehrere Wohnsitze, Zugehörigkeiten und kontinuierliche Mobilität während des Lebensverlaufes verbunden, wie es zum Teil bei Ski- und Tauchlehrern, Animateuren, Kreuzfahrern oder Kletterern zu beobachten ist (vgl. Cohen/Duncan/Thulemark 2015, S. 158 ff.).

${ }^{3}$ Die (Verkehrs-) Mobilität ,(...) weist bereits auf die enge Beziehung zum Verkehr hin, ist aber eine Krampflösung. Sie wird im Bereich der Verkehrswissenschaft auch immer nur dann verwendet, wenn man bewußt die übrigen (...) aufgezeigten Mobilitätskategorien ausschließen will. Im allgemeinen erfolgt dieser Ausschluß unter Verkehrswissenschaftlern jedoch stillschweigend, so daß dann - statt von Verkehrsmobilität - wiederum nur von Mobilität (aber eben im eingeschränkten Sinne der Verkehrsmobilität) die Rede ist" (Cerwenka 1999, S. 35). So wird auch im Rahmen der vorliegenden Untersuchung verfahren. 
Abb. 1 Mobilität als Gegenstand der Forschung. (Quelle: Vgl. Groß 2005, S. 38)

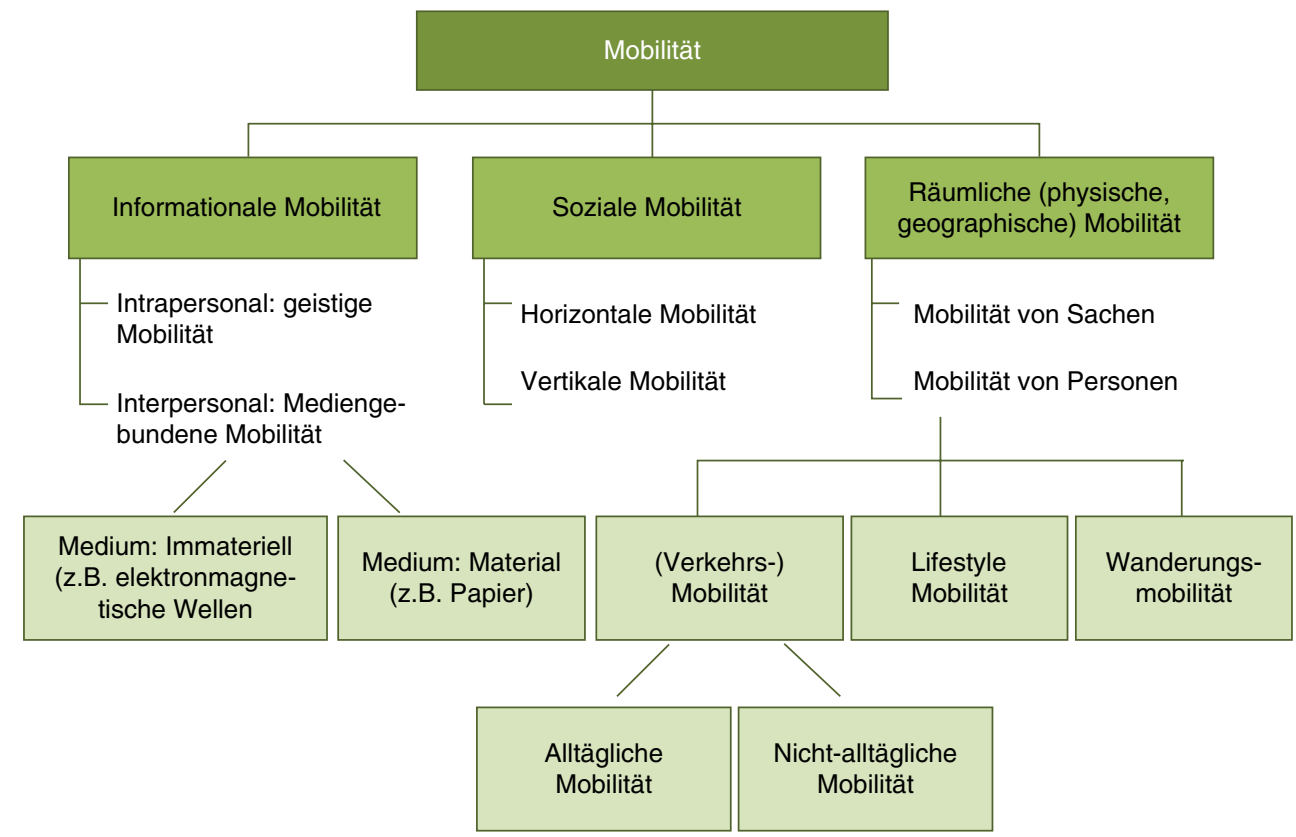

„Point of Interest“ (Pol) - also z. B. den Fragen, warum an einem bestimmten Ort angehalten wird, was dort unternommen wird, ob Ausgaben getätigt werden oder welche weiteren Entscheidungen vor Ort getroffen werden - trotz erster Untersuchungen (z. B. Kazig/Popp 2011 und die dort genannten Quellen) vergleichsweise wenig Aufmerksamkeit (vgl. Millonig/Gartner 2007, S. 31; Nielsen/Blichfeldt 2009, S. 2; Spangenberg 2014, S. 317 f.).

Ziel ist es, mithilfe des Einsatzes einer neuen Software Pol-genaue Informationen zu generieren, die sich einer vorhandenen Methode, des GPS-Tracking, zur Erfassung des (touristischen) Mobilitätsverhaltens bedient, diese aber um neue Verfahrensweisen bei der Erfassung und Aufbereitung der Daten ergänzt. Hierzu werden in Kap. 2 zunächst die vorhandenen Ansätze auf Potenziale und Begrenzungen hin gesichtet und es wird unter anderem der Frage nachgegangen, inwiefern bei den bestehenden Methoden das Verhalten der Probanden, ihre Handlungen vor Ort, die zugrunde liegende Motivation und die Zufriedenheit mit bzw. bei den besuchten Pol erfasst werden können. Schwerpunkt der Betrachtung sind hierbei die GPS-TrackingMethoden, die im Outdoor-Bereich genutzt werden können.

Mit der eigens entwickelten Software, einer „Applikation“, die in Kap. 3 des Beitrages vorgestellt wird, soll eine Analyse auf einer Ebene ermöglicht werden, die Individuum (z. B. Motivation, Zufriedenheit, soziodemographische Merkmale, Reiseverhalten), Handlung (im Sinne von Aktivität am Pol) und Mobilität im Raum vereint (vgl. hierzu auch Weber/Bauder 2013, S. 100). Anschließend werden in Kap. 4 empirische Ergebnisse aus einem Fallbeispiel im Harz dargestellt, um zu skizzieren, welches Potenzial die hier vorgestellte Kombination von Instrumenten aufweist. Hierbei handelt es sich um eine von mehreren durchgeführten Erhebungen, die an sechs Erhebungstagen vom 18. Mai bis 13. Juni 2013 am Brocken im Harz stattfand. An den Untersuchungstagen konnten mehr als 100 GPS-Logger auf dem Brockenplateau an Wanderer ausgegeben werden; nach Abschluss der Wanderung fanden Face-to-Face-Befragungen statt. Eine zusammenfassende Darstellung im Kap. 5 fasst Limitierungen, Nutzen und die Potenziale der vorgestellten Applikation zusammen.

\section{Methoden zur Erfassung des Mobilitätsverhaltens}

Erste Studien zur Erfassung des Mobilitätsverhaltens finden sich bereits in den 1950er- und 1960er-Jahren, wie die verdeckten Beobachtungen und Befragungen des Instituts für Demoskopie aus Allensbach (vgl. Institut für Demoskopie 1956, S. 2 ff.). Eine erste Blüte haben derartige Untersuchungen in den 1970er- und 1980er-Jahren, vor allem in der (Zeit-) Geographie mit ihren theoretischen und analytischen Studien (z. B. Hägerstrand 1970; Thrift 1977; Carlstein/Parkes/Thrift 1978). Bis Ende der 1990er-Jahre wurden die Daten für empirische Arbeiten weitestgehend über Interviews (z. B. Higham/Holt/Kearsley 1996), Beobachtungen (z. B. Hartmann 1988; Murphy 1992; Keul/ Kühberger 1996), „Mental Maps“ (z. B. Downs/Stea 1982) oder Tagebücher (z. B. Fennell 1996; Thornton/Williams/ Shaw 1997) erhoben. Mit Ende des 20. Jahrhunderts bzw. Beginn des 21. Jahrhunderts ergeben sich neue Möglichkeiten der Mobilitätserfassung mithilfe von GSM, UMTS, Bluetooth, GPS, „Photo-Sharing-Communities“, Scanner, Sensoren oder Videoanalysen. Zur Datenverdichtung und Verbesserung der Qualität der Ergebnisse werden diese 
Methoden in der heutigen Mobilitätsforschung häufig auch in Kombination verwendet.

Die folgende Abb. 2 gibt einen Überblick über die wichtigsten Methoden zur Erfassung des Mobilitätsverhaltens der letzten Jahrzehnte. Im Anschluss wird das GPS-Tracking in seinen verschiedenen Varianten der Kombination mit anderen Methoden skizziert. Es geht dabei vorrangig darum, den Kerninhalt, die Potenziale und Begrenzungen darzustellen sowie zu klären, wo eine neue Anwendung zur Weiterentwicklung bei der Untersuchung (touristischer) Mobilität ansetzen kann.

Es existieren mehrere Ansätze, um GPS-TrackingDaten mit weiteren Ergebnissen anzureichern, wovon im Folgenden eine Auswahl vorgestellt wird. GPS-Tracking wird vor allem dann angewendet, wenn die zu eruierende Fragestellung beispielsweise das Verhalten der Probanden an einem bestimmten Wegepunkt (Pol), die Ermittlung der Zufriedenheit der erfassten Personen mit besuchten Attraktionsfaktoren, Restaurants, Kultureinrichtungen o. ä. bzw. die der zurückgelegten Strecke zugrunde liegende Motivation betrifft.

So haben im Leipziger Zoo die Besucher per Knopfdruck auf dem ausgegebenen GPS-Gerät markiert, ob ihnen Stellen besonders gefallen oder nicht so gut gefallen haben - weitergehende Informationen (z. B. zu Attraktivitätsund Störfaktoren oder den genauen Tätigkeiten) zu den markierten Orten wurden nicht erhoben (vgl. Czaplicki 2009, S. 76 ff.).

Ein weiterer Ansatz ist der Prompted Recall Survey. Hier wurden GPS-Tracker mit Funktionstasten ausgestattet und der GPS-Track wurde nach Abschluss der zurückgelegten Strecke in einer gedruckten Karte angezeigt, sodass er als Gedankenstütze zum Einholen weiterer Informationen diente. Dazu gehörten das Aufdecken von möglichen Fehlaufzeichnungen im GPS-Track, das Identifizieren von Pausenzeiten und deren Gründe sowie Informationen zum verwendeten Verkehrsmittel und der Reisebegleitung. Erste Erfahrungen zeigen, dass beim Umgang mit diesen Geräten
Bedienungsschwierigkeiten wie fehlerhafte bzw. unvollständige Eingaben durch die Probanden auftraten. Zudem achteten sie nicht regelmäßig auf den Akkustand und empfanden die Mitnahme eines zusätzlichen Gerätes als unangenehm (vgl. Bachu/Dudala/Kothuri 2001; Stopher/ Prasad/Zhang 2010).

Ein anderer Ansatz besteht in der Verknüpfung von GPSTracking mit der Visitor Employed Photography (VEP) und einer standardisierten Befragung (vgl. Cherem/Driver 1983, S. 66 f.). Zur Erhebung der Bild- und GPS-Daten wurden die Probanden aufgefordert, entlang ihres Weges alles zu fotografieren, was sie als schön oder störend empfinden. Die von den Probanden angegebenen Bildmotive und Begründungen für jedes Foto wurden einer Inhaltsanalyse unterzogen und in ihrer Häufigkeit bestimmt. Mittels lagebezogener Analyseverfahren wurden für alle Aufmerksamkeitspunkte „CP strength“-Werte bestimmt. „CP strenght“ definiert den prozentualen Anteil der Probanden, die von einem bestimmten Punkt eine Aufnahme gemacht haben, in Relation zu allen Probanden, die diesen Punkt passierten. Ebenfalls wurden soziodemographische Daten erhoben und die mitgeführten GPS-Datenlogger ausgelesen. Als Nachteil wird der vergleichsweise hohe technische und personelle Aufwand angesehen (vgl. Graaff 2014).

Eine andere Möglichkeit besteht darin, die Ergebnisse aus dem GPS-Tracking mit einem standardisierten Fragebogen zu kombinieren. Van der Spek/van Schaick/de Bois et al. (2009) haben im Rahmen ihrer Untersuchung von Fußgängern in drei europäischen Stadtzentren neben der Ausgabe von GPS-Loggern eine Face-to-Face-Befragung durchgeführt und anschließend die Daten in die Statistik- und Analyse-Software SPSS integriert und eine Verknüpfung mit dem Geoinformationssystem ArcGIS hergestellt. Auch andere Wissenschaftler haben erste Erfahrungen mit der Kombination beider Methoden gesammelt (siehe dazu unter anderem Arrowsmith/Chhetri 2003; Shoval/Isaacson 2010; Bauder 2011; Bauder 2012; Weber 2012a; Weber 2012b).

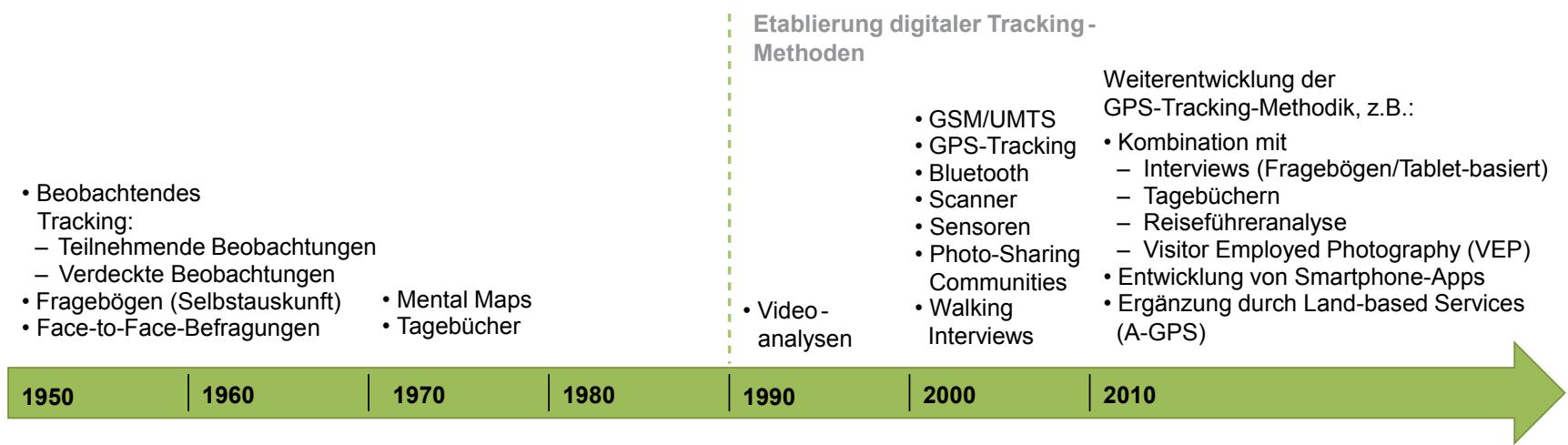

Abb. 2 Entwicklung der Methoden zur Erfassung des Mobilitätsverhaltens. (Quelle: Eigene Darstellung, in Anlehnung an Weber/Bauder 2013, S. 108) 
Eine Herausforderung besteht allerdings in der Zuordnung der Pol, um die gewünschten ortsgenauen Informationen generieren zu können (vgl. Czaplicki 2009; Bauder 2011; McKercher/Shoval/Ng et al. 2012; Weber 2012a; Weber 2012b; Spangenberg 2014). Insbesondere in Bereichen, in denen mehrere Attraktionen dicht beieinanderliegen (z. B. an einem Marktplatz mit Cafés, Rathaus, Brunnen und mehreren Sehenswürdigkeiten nebeneinander), ist die genaue Zuordnung schwierig. Eine mögliche Lösung wird mithilfe der sogenannten „Bufferanalyse“ gesucht, bei der digitalisierte Sehenswürdigkeiten mit den GPS-Daten abgeglichen werden, um herauszufinden, ob sich die Probanden in der Nähe der Sehenswürdigkeit befunden haben oder nicht (vgl. Weber 2012b, S. 196). ${ }^{4}$ Aber auch hier gibt es Probleme bei der Zuordnung. Beispielsweise stellt sich die Frage, ab wie viel Metern Annäherung zu einem Pol davon ausgegangen werden kann, dass dieser bewusst besucht bzw. gesehen wird. „Die Bufferanalyse ist kein vollständiger Nachweis dafür, dass der Träger des GPSGerätes die Sehenswürdigkeit wirklich gesehen oder wahrgenommen, geschweige denn diese willentlich aufgesucht hat. Möglicherweise ist die Person nur daran vorbeigelaufen, um vielleicht einen anderen Ort aufzusuchen. Dennoch besteht mittels dieser Bufferanalyse zumindest die Möglichkeit, Tendenzen aufzuzeigen, wo sich Reisende besonders konzentriert aufhalten bzw. woran sie vorbeilaufen “ (Weber 2012b, S. 198).

An dieser Stelle blieb die Bufferanalyse ohne ein konkretes Ergebnis (vgl. Bauder 2011; Weber 2012b). Möglichkeiten, diese Problematik zu umgehen, sind direkte Befragungen zu den besuchten Wegepunkten. So können z. B. die Wegepunkte im Nachhinein gemeinsam mit den Probanden mithilfe von Google Earth durchgegangen und hierzu Fragen gestellt werden (z. B. zur Beschilderung, zu den Gründen für die Auswahl der besuchten Pol, zu den Handlungen), um unter anderem herauszufinden, welche Pol besucht wurden, wie viel Zeit am jeweiligen Pol verbracht wurde und welche Serviceleistungen in Anspruch genommen wurden (vgl. Edwards/Griffin/Hayllar et al. 2009; Edwards/Griffin 2013).

Zusammenfassend lässt sich in Bezug auf die Ergebnisse vorangegangener Forschungsprojekte festhalten, dass die Ermittlung bzw. Erhebung ortsgenauer Informationen zu Wegepunkten (z. B. verbrachte Zeitdauer am Wegepunkt, in Anspruch genommene Leistungen, Attraktivitäts- und Störfaktoren) zwar möglich ist, aber noch selten eingesetzt wird. Je nach Forschungsfrage und Ansatz der Untersuchung können die einzelnen Methoden sinnvoll und ziel-

\footnotetext{
${ }^{4}$ Die als Sehenswürdigkeiten definierten Orte wurden als proportionaler Kreis symbolisiert, wobei die Anzahl der Besucher je Sehenswürdigkeit die Größe der Kreissignatur bedingte (je mehr Besucher, desto mehr GPS-Punkte und somit größere Kreissignatur) (vgl. Bauder 2011, S. 43 f.).
}

führend sein. In Anknüpfung an die bisherigen Ergebnisse scheint die Entwicklung einer Anwendung angebracht, die eine automatische Verknüpfung von Wegepunkten und Motivationen, Aktivität und Verhaltensweisen auf dem zurückgelegten Weg und am Pol sowie die Zufriedenheit mit verschiedenen Aspekten am Pol ermöglicht. Dies wurde mittels einer Android-basierten Applikation für Tablet-PCs umgesetzt, mit deren Hilfe die Interviews computergestützt durchgeführt und die Daten in eine externe onlinebasierte Datenbank eingespeichert werden können.

\section{Neue Anwendung zur Erfassung des Mobilitätsverhaltens mittels GPS-Technik}

Im Folgenden wird die entwickelte Software vorgestellt, die mittels Tablet-Computer und einer eigens programmierten Befragungssoftware (Applikation auf Android-Basis) die oben genannten Daten erhebt. Die nachfolgende Abb. 3 fasst die vier wichtigsten Ziele und Elemente der neuen GPS-Tracking-Anwendung zusammen, wobei die Ziele 3 und 4 die eigentlichen Neuerungen ausmachen.

\subsection{Implementierung des Befragungssystems und Auswertung der Daten}

Für die Erfassung des Mobilitätsverhaltens wurden GPSLogger genutzt, die den Austausch der GPS-Daten per Speichermedium (SD-Card), USB-Schnittstelle oder Funk (z. B. Bluetooth) unterstützen. ${ }^{5}$ Das Columbus-V-900-Gerät verfügt sowohl über eine Bluetooth-Schnittstelle als auch über einen Micro-SD-Speicherslot zum Einschub der Speicherkarte. Zudem verfügt es über eine Speicherkapazität von bis zu 25 Mio. Wegepunkten, eine Akkulaufzeit bei Dauerbetrieb von bis zu $24 \mathrm{~h}$, einen MTK-2-Chipsatz mit 66 parallelen Satellitenverfolgungskanälen, eine interne Keramik-Patchantenne mit einer Empfindlichkeit von $-165 \mathrm{dBm}$ und unterstützt die satellitenbasierten Ergänzungssysteme WAAS/EGNOS/MSAS ${ }^{6}$. Die Wahl eines geeigneten Tablet-Computers richtete sich vor allem nach den verfügbaren Schnittstellen zur direkten Anbindung an die GPS-Logger (Micro-SD-/USB-Schnittstelle). Zudem sollte das Gerät WLAN-fähig sein, 3G-Netze

\footnotetext{
${ }^{5}$ Eine in Betracht gezogene Nutzung von Smartphones für die Erfassung der Wege wurde verworfen, da Smartphones bei Projektstart im Jahr 2012 noch eine zu geringe Verbreitung hatten (2012: 29\% und 2014: 50\%) (vgl. Lopez 2014, S. 4).

${ }^{6}$ WAAS (=Wide Area Augmentation System), EGNOS (=European Geostationary Navigation Overlay Service) und MSAS (=Multifunctional Satellite Augmentation System) nutzen zusätzliche Informationen, die von geosynchronen (meistens geostationären) Satelliten ausgestrahlt werden, um die Zuverlässigkeit, Genauigkeit und Verfügbarkeit der Positionsbestimmung durch GPS zu erhöhen.
} 

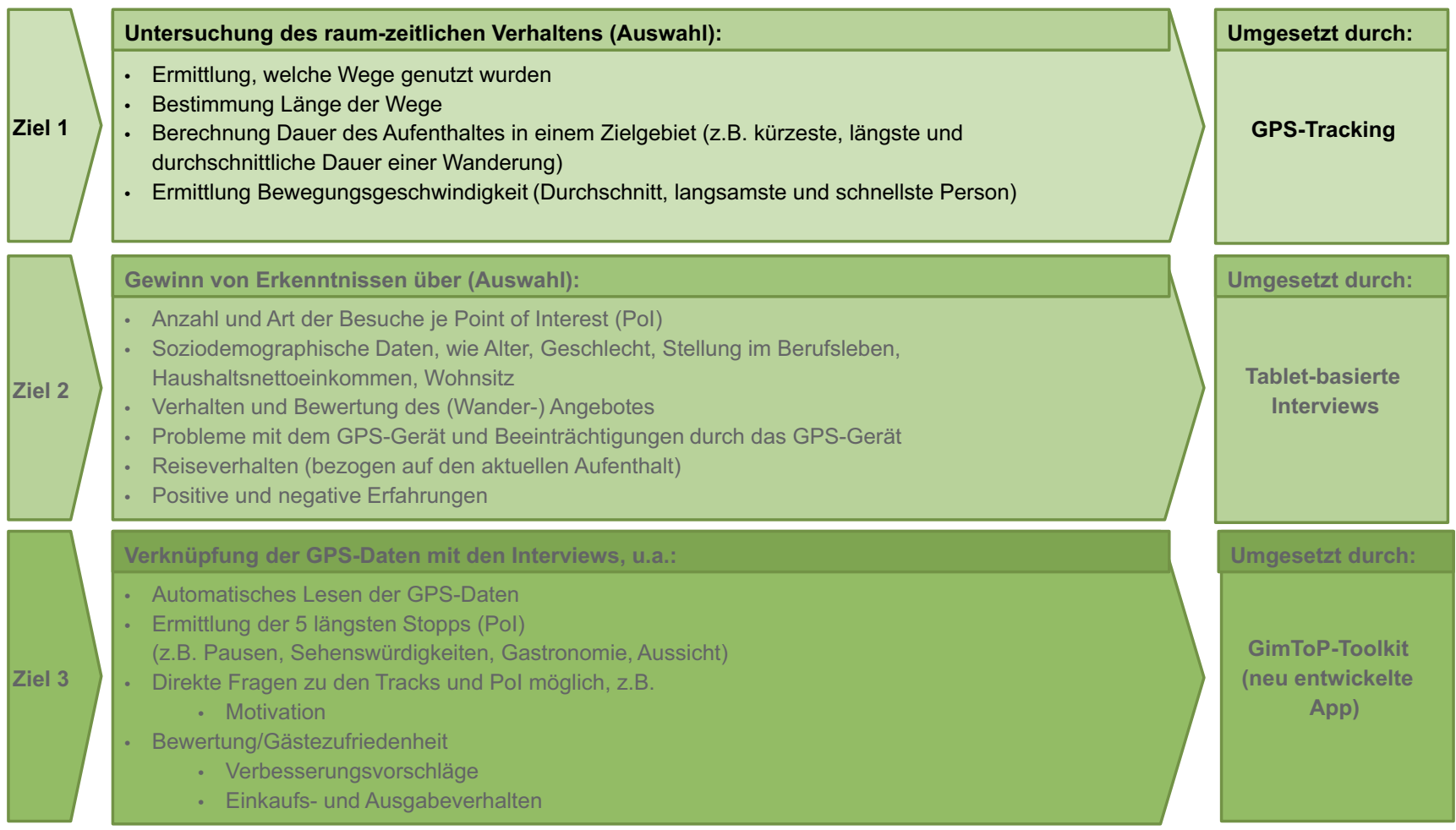

Verknüpfung der Mobilitätsdaten mit den Befragungsergebnissen:

Umgesetzt durch:

Ziel 4

u.a. mit soziodemographischen, reisespezifischen Daten und Wandereigenschaften

- Automatisierung der Datenübertragung

Abb. 3 Ziele und Elemente von GPS-Tracking-Anwendungen. (Quelle: Eigene Darstellung)

(mit erweiterter Funktionalität besonders im Bereich Multimedia) unterstützen und auf einem Android-Betriebssystem laufen. ${ }^{7}$ Bei den erhältlichen Tablets traf das Asus EeePad Transformer TF300 TG diese Anforderungen am besten und wurde daher verwendet.

\subsection{Das GimToP Toolkit (GTK)}

Mithilfe des GimToP-Toolkit (GTK) lassen sich die GPSTracking-Komponenten mit den Inhalten der mündlichen Interviews kombinieren (vgl. Abb. 4). Das GTK besteht

\footnotetext{
${ }^{7}$ Ziel sollte es sein, eine Applikation zu entwickeln, die auf unterschiedlichen mobilen Endgeräten eingesetzt werden kann. Daher sollte ein mobiles Betriebssystem verwendet werden. Zum Zeitpunkt der Entwicklung gab es zwei Marktführer für mobile Betriebssysteme: iOS (ca. 17\%) und Android (ca. 70\%) (vgl. IDC 2015). Die Entscheidung für Android hatte sechs Gründe: 1) Die größere Auswahl an Endgeräten. 2) Die Android-Geräte waren bzw. sind günstiger als die von Apple. 3) Als Schnittstelle für den Import der GPS-Tracks wurde ein SD-Kartenslot benötigt. Diesen hatte das iPad/ iPhone nicht integriert. 4) Zusätzliche Kosten für die Entwicklung der App (Apple-Developer-Account und Apple Rechner notwendig) sollten vermieden werden. 5) Entsprechende Frameworks für die Programmierung sollten verfügbar sein. 6) Apple setzte bzw. setzt auf eine ältere, weniger verbreitete Programmiersprache (Objective C).
}

aus einer mobilen Anwendung für die Datenerfassung und einem Server für die Verarbeitung der Daten in einer serviceorientierten Architektur.

\subsubsection{Datenerfassung}

Die mobile GTK-Anwendung wird für die digitale Erfassung der Erhebungsdaten verwendet. Im Unterschied $\mathrm{zu}$ bestehenden Anwendungen bietet sie die Möglichkeit, bereits während der Befragung raumbezogene Daten (GPS-Tracks) anzuzeigen, zu verarbeiten und sie direkt in Befragungen zu integrieren. Zudem ist es mithilfe der Anwendung möglich, Informationen zur Aktivität an einem bestimmten Wegepunkt zu erfassen und so zu ermitteln, welche Entscheidungen hier getroffen wurden.

Die GTK-Anwendung besteht vor allem aus dem sogenannten „Analyzer" und einem text- und kartenbasierten Befragungsmodul. Die Aufgaben des ersten Moduls sind der Import der aufgezeichneten Tracks und ihre Bearbeitung im Sinne einer Fehleranalyse und Korrektur vorhandener Ausreißer. Letztere werden automatisch durch den Vergleich der prozentualen Verschiebung der Höhenund Geschwindigkeitsangaben zu den vorherigen und nachfolgenden Wegepunkten in einer definierten Zeitperiode 
Abb. 4 Architektur GimToP Toolkit (GTK). (Quelle: Vgl. Spangenberg 2014)

\section{Datenerfassung}

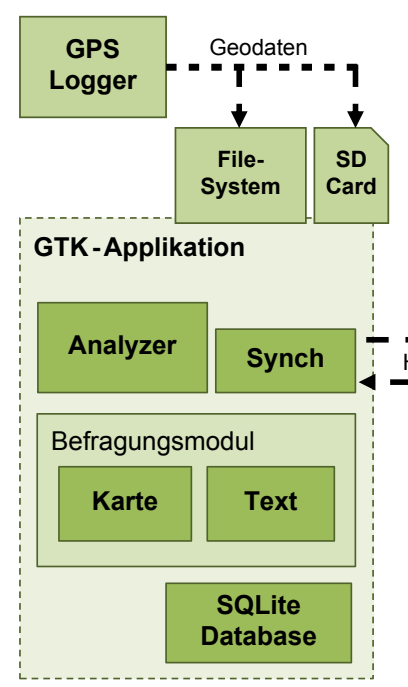

ermittelt, angezeigt und per Hand gelöscht. Das textbasierte Befragungsmodul ist das digitale Gegenstück zum Papierfragebogen. Aufgrund der unmittelbaren Digitalisierung der Daten ist die zeitliche Nachbearbeitung der Daten weniger aufwendig als bei einem Papierfragebogen - eine Prüfung auf logische Konsistenz, Eingabefehler u. ä. wird aber auch hier durchgeführt.

Mithilfe des kartenbasierten Moduls können die Wegestrecken der Probanden dargestellt werden. Die GTKAnwendung importiert und visualisiert die Tracks in einer interaktiven Karte, zeigt die Verweilzeiten an den Streckenabschnitten an (inklusive einer Positionsanzeige der GPSKoordinaten) und bindet zudem weitere statistische Daten wie Informationen zur zurückgelegten Distanz (in km), zur dafür benötigten Zeit (in h) und zur erreichten Durchschnittsgeschwindigkeit (in $\mathrm{km} / \mathrm{h}$ ) ein. Letztere wird einerseits als Weg durch Zeit berechnet (= Durchschnittsgeschwindigkeit inklusive der Pausen) und andererseits mithilfe des SMoT-Algorithmus (,stops and moves of trajectories") berechnet (= Durchschnittsgeschwindigkeit exklusive Pausen), welcher durch Alvares/Bogorny/Kuipers et al. (2007) entwickelt wurde (Näheres dazu siehe unten). Darüber hinaus wurde eine Trackbereinigungsfunktion inkludiert, indem ein eigener Algorithmus implementiert wurde, der alle Punkte des Tracks (des Weges durch die Landschaft bzw. des „Kurses“ auf der Landkarte) durchläuft und die Geschwindigkeits- und Höhensprünge aufruft und bereinigt (vgl. Spangenberg 2014).

Als Kartenbasis wird OpenStreetMap ${ }^{8}$ (OSM) genutzt. Im Vergleich $\mathrm{zu}$ anderen Diensten ermöglicht OSM die

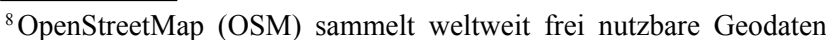
über Straßen, Eisenbahnen, Flüsse, Wälder, Häuser usw. Diese sind als Rohdaten oder Karten abrufbar. Das freie Projekt OSM finanziert sich durch Spenden (s. http://www.openstreetmap.de/).
}

\section{Datenverarbeitung Datenanalyse}

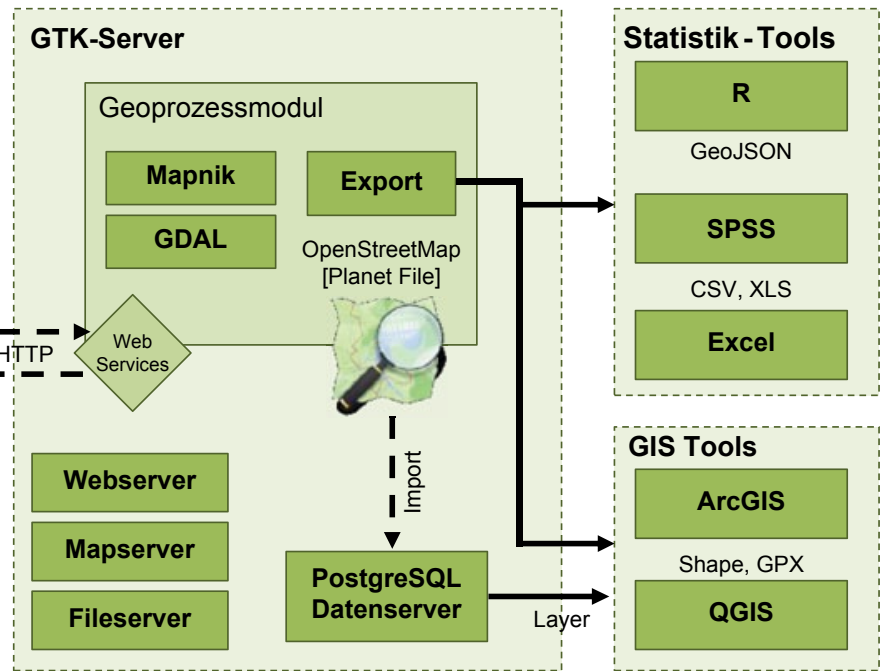

kostenlose Nutzung der Rohdaten für die Umsetzung der eigenen Zwecke, steht uneingeschränkt zur Verfügung und verfügt über verschiedene Kartenstile. Des Weiteren werden die weltweiten OSM-Rohdaten auf XML-Basis wöchentlich aktualisiert. Positiv unter dem Gesichtspunkt der grundsätzlichen Datenverfügbarkeit ist es, dass jeder Daten, die er oder sie für wichtig hält, bei OSM eingeben, verändern, verbessern oder auch löschen kann. Hierfür ist nur eine vorherige Anmeldung bzw. Registrierung bei OSM notwendig. Darauf ist gleichzeitig auch ein in methodischer Hinsicht gravierender Nachteil der Daten zurückzuführen, da bei ca. 5 Mrd. eingepflegten Datenpunkten und mehr als 2,3 Mio. registrierten Nutzern (Stand Ende 2015) nicht für eine gleichbleibend hohe Qualität und Genauigkeit der Rohdaten gesorgt werden kann (vgl. Neis 2008, S. 19 ff.; Ramm/Topf 2010, S. 19 ff.; OSM 2015a).

Die Karten und Pol von OSM lassen sich über eine eigens implementierte Schnittstelle mit der App synchronisieren (= „Synch“ in Abb. 4), und alle Daten werden auf einer lokalen SQLite-Datenbank (interner Speicher des GPS-Gerätes) gespeichert. Somit ist sichergestellt, dass die jeweilige Karte sowohl online als auch offline verwendet werden kann, was vor allem in ländlichen Regionen, die nicht flächendeckend über mobile Internet-Verbindungen verfügen, sinnvoll ist.

Weiterhin werden neben den raum-zeitlichen Bewegungsdaten auch die besuchten Pol mithilfe eines Pausensymbols in der Karte dargestellt (vgl. Abb. 5). Hierfür wird der von Alvares/Bogorny/Kuipers et al. (2007) entwickelte SMoTAlgorithmus genutzt. Er erfordert eine Parametrisierung der räumlichen und zeitlichen Dimensionen einer Pause, die durch eine minimale Verweilzeit $t$ in einem vorbestimmten Radius $r$ definiert ist. Den vorliegenden Studien sind keine einheitlichen Hinweise auf einen dafür vorzugebenden Schwellenwert zu entnehmen - so wurden bisher beispielsweise Werte zwischen einer Minute und zwei Minuten 
Abb. 5 Befragung mithilfe des kartenbasierten Befragungsmoduls. (Quelle: Eigene Darstellung)

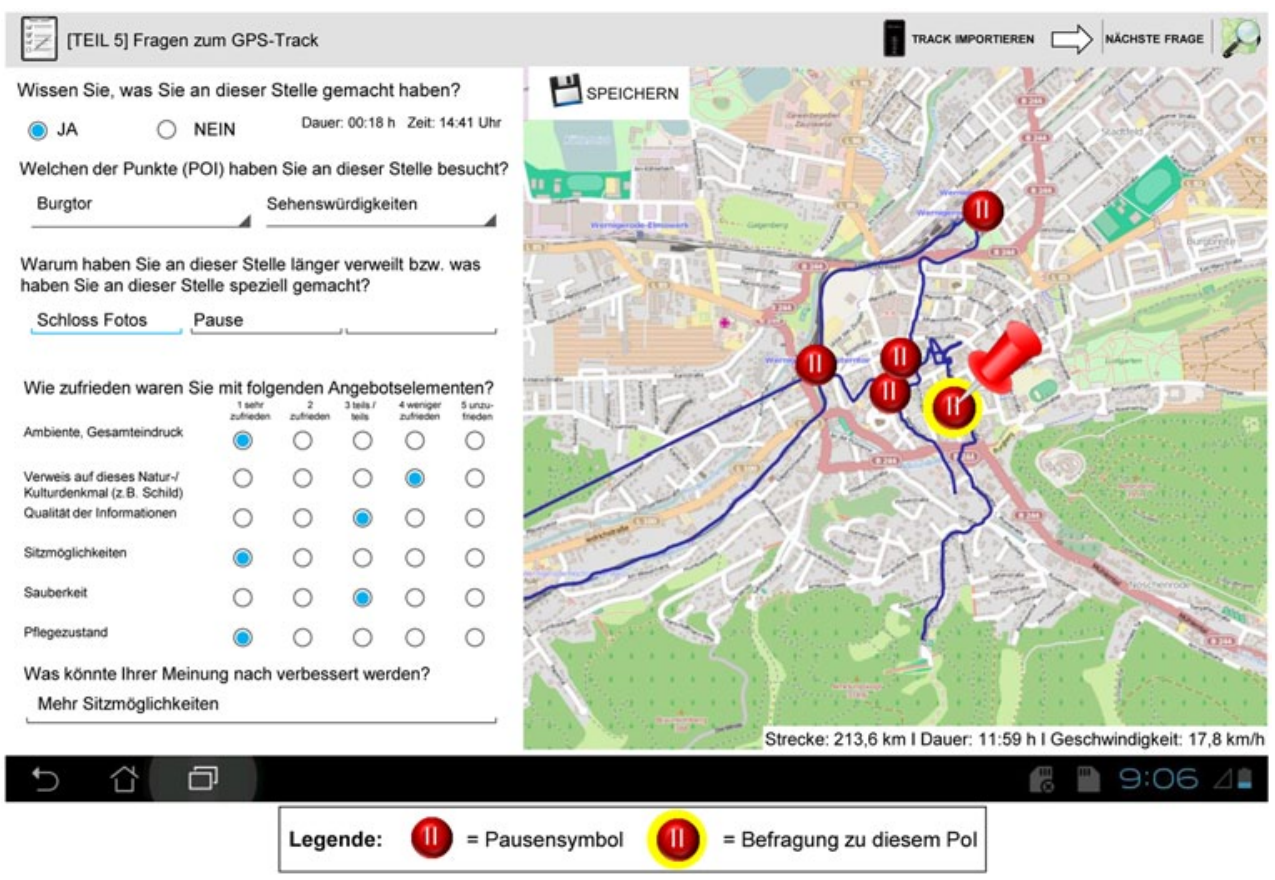

angesetzt (vgl. Alvares/Bogorny/Kuipers et al. 2007; Clifford/Zhang/Stopher 2008, S. 2). Der GTK-Analyzer definiert in der hier vorgestellten Fallstudie eine Pause aus pragmatischen Überlegungen bei $t=3 \mathrm{~min}$. und einem Radius $r=50 \mathrm{~m}$, um die Anzahl der angezeigten Pol überschaubar zu halten.

$\mathrm{Da}$ die Befragungsdauer einen Zeitraum von ca. 15-20 min nicht überschreiten sollte, wurde zu einer begrenzten Anzahl an Pol befragt. Bei einem Vortest (,,pretest“) hatte sich herauskristallisiert, dass fünf Pol im genannten Zeitraum gut erfassbar sind. Hinzu kommt, dass diese Pol für eine Befragung in der Regel besonders relevant sind, da Personen dazu neigen, sich länger an Orten aufzuhalten, die sie wirklich interessieren bzw. wenn die Notwendigkeit besteht, sich auszuruhen oder Serviceleistungen (z. B. Restaurant, Imbiss) in Anspruch zu nehmen. Daher wurden die fünf am längsten besuchten Pol mithilfe eines gelben Rahmens hervorgehoben. Der Interviewer hat die Möglichkeit, auf das Pausensymbol oder einen anderen Ort auf der Karte zu klicken, und es wird daraufhin ein Dialogfenster angezeigt. Um zu ermitteln, warum beispielsweise dieser Pol besucht wurde, welche spezifischen Aktivität an diesem Pol durchgeführt wurden, wie es um die Zufriedenheit oder um Möglichkeiten zu Verbesserungen steht, werden diese Pol von der Applikation mit Fragen (Textfeldern) verknüpft.

In diesem Zusammenhang ist die Frage der eindeutigen Zuordnung des an dieser Stelle aufgesuchten Pol hervorzuheben. Um die Auswahl zu erleichtern, wird automatisch eine Suche aller Pol im Umkreis von $50 \mathrm{~m}$ durchgeführt und die Ergebnisse in einer Auswahlliste angezeigt. Der konkrete Pol kann dann zugeordnet werden oder eine manuelle Ein- gabe der Pol erfolgen, sofern der Pol nicht in der Auswahlliste angezeigt wird oder es sich um eine Kombination von zwei oder mehr Pol handelt. Dieses manuelle „Einchecken“ von Pol ist vor allem dann wichtig, wenn Befragungen an Orten durchgeführt werden, die eine hohe Anzahl unterschiedlicher Pol in einem kleinen Radius aufweisen. Ohne dieses Vorgehen ist die Pol-Auswertung der GPS-Daten nahezu unmöglich, da die Genauigkeit der GPS-Logger zwischen 10 und $30 \mathrm{~m}$ liegt und sich in diesem Radius in städtischen Kontexten mitunter zahlreiche Pol befinden können. Diese manuelle Festlegung des Pol und die punktgenaue Befragung zu einzelnen Pol ist eine Neuerung, die so in keinem der mehr als 100 ausgewerteten wissenschaftlichen Beiträge dargestellt wurde (siehe hierzu Kap. 2).

Nach Abschluss der Befragung können die erhobenen Daten per WLAN, 3G-Netz oder auch manuell auf einen externen Server gespeichert werden.

\subsubsection{Datenverarbeitung}

Der Linux-basierte GTK-Server sorgt für die Bereitstellung von Geodaten wie Karten und Pol für die mobile GTK-Anwendung. Die zugrunde liegenden Daten können durch Webservice-Schnittstellen abgerufen werden und werden von Open Street Map (OSM) generiert. Mit einer unkomprimierten Dateigröße von über $400 \mathrm{~GB}$ beansprucht der Import der Daten allerdings viel Rechenzeit und -aufwand. Aus diesem Grund verwendet der GTK-Server einen kostenlosen Service des Unternehmens Geofabrik GmbH, welcher den „OSM planet file“ in einzelne kleinere Dateien teilt, also z. B. in Regionen, Kontinente, Staaten und Länder. Die reduzierten OSM-Rohdaten lassen sich dadurch leichter 
in die PostgreSQL-Datenbank des GTK-Servers importieren und können mithilfe der GDAL-Tools (Geospatial Data Abstraction Library) dargestellt werden. Anschließend wird das Open-Source-Toolkit „Mapnik“ von Artem Pavlenko angewandt, um aus diesen Daten Karten zu erstellen. Die Karten werden auf den GTK-Dateiserver gespeichert und können im internen Speicher der GTK-Anwendung auch für die Offline-Nutzung heruntergeladen werden.

Die mehr als 1.000 bei OSM vorhandenen Pol-Kategorien, die über sogenannte „Map Features“ beschrieben werden, einer Kombination von Schlüssel- und Werteelementen („Attribute“), werden den in der Tabelle 1 dargestellten Kategorien zugewiesen (vgl. OSM 2015b). Diese wurden von den Autoren in Anlehnung an das touristische Angebot einer Destination (vgl. z. B. Inskeep 1991; Freyer 2015, S. 323) und beruhend auf der Klassifikation der Wirtschaftszweige des Statistischen Bundesamtes (2007) entwickelt, sodass sich 405 Attribute, das heißt 405 PolKategorien durch OSM ergeben, welche den neun Kategorien zugeordnet wurden (vgl. Tab. 1). Allerdings lässt die GTK-Anwendung es zu, dass ein Pol auch keiner der neun Kategorien zugeordnet wird und somit eine manuelle Eingabe möglich ist. Je nach Anzahl der erfassten Pol können die einzelnen Kategorien allein oder mehrere Kategorien zusammen genauer betrachtet werden.

Die zweite Aufgabe des GTK-Servers besteht in der Aggregation und Verarbeitung der Befragungsdaten, die von der mobilen GTK-Anwendung gesammelt werden.
Der GTK-Server stellt dazu Schnittstellen zum Import der Daten zur Verfügung. Die GPS-Tracks werden in einer PostgreSQL-Datenbank gespeichert und automatisch mit den textbasierten Befragungsdaten aggregiert. Zwischen der Datenerfassung mit der mobilen GTK-Anwendung und der Datenanalyse (z. B. mit GIS) sind keine weiteren Zwischenschritte erforderlich, was den Arbeitsaufwand bei GPSbasierten Befragungen erheblich reduziert. Die Auswertung der Umfrage ist nicht Teil des GTK. Zu diesem Zweck kommen bestehende statistische oder geobasierte Analyseanwendungen zum Einsatz (z. B. Excel, GIS-Tools, R, SPSS). Diese bieten eine breite Palette von Möglichkeiten, um Daten mit verschiedenen Anwendungen zu analysieren.

\section{Datenerhebung zum Mobilitätsverhalten von Wanderern am Brocken}

Der Harz erstreckt sich über die drei Bundesländer Niedersachsen, Sachsen-Anhalt und Thüringen und hatte im Jahr 2014 ca. 6,42 Mio. Übernachtungen (vgl. Statistisches Bundesamt 2015, S. 12 f.). Im Harz stehen das Wandern, das Naturerleben, die Erholung sowie der Besuch kultureller Sehenswürdigkeiten für die meisten Besucher im Mittelpunkt des Aufenthaltes. Der Brocken ist hierbei mit etwa 600.000 Besuchern pro Jahr das meist besuchte Ausflugsziel (vgl. inspektour GmbH/ift GmbH 2012, S. 32 ff.; Job/ Woltering/Schamel et al. 2014, S. 75).

Tab. 1 Aus der OSM-Kartenfunktion abgeleitete und für die GTK-Anwendung definierte Pol-Kategorien. (Quelle: Eigene Darstellung, in Anlehnung an Inskeep 1991; Statistisches Bundesamt 2007; Freyer 2015, S. 323)

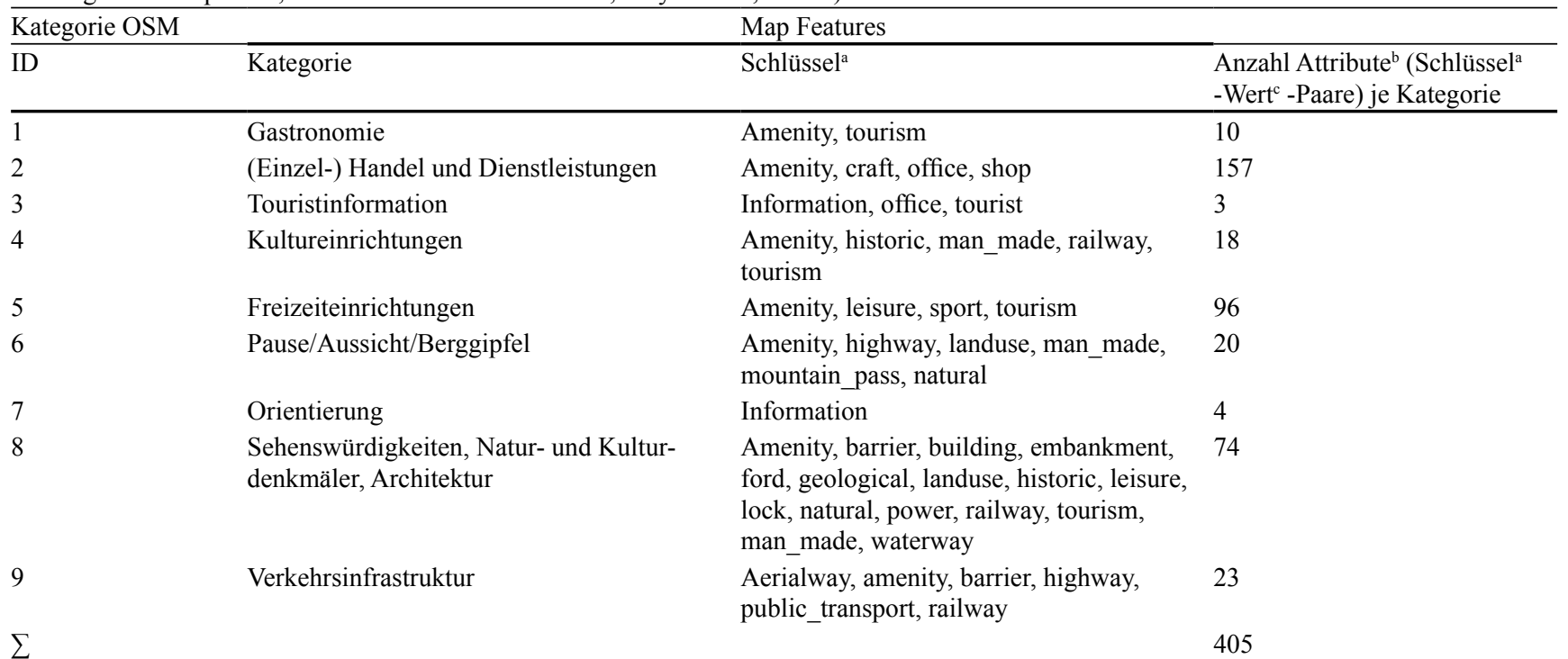

${ }^{a}$ Der Schlüssel bestimmt die Art der beschriebenen Eigenschaft (z. B. highway =*). Kurz: Er macht aus einer OSM-Linie eine Straße.

${ }^{b}$ In der OSM-Dokumentation werden Attribute als schlüssel = wert dargestellt. Schlüssel und Werte sind freie Textfelder, die jedoch nach bestimmten Konventionen zu verwenden sind.

${ }^{c}$ Der Wert beschreibt die Eigenschaft dann genauer (z. B. highway = motorway oder track). Kurz: Er stuft die Straße als Autobahn oder als Feldweg ein. 
Tab. 2 Soziodemographische Merkmale der Probanden. (Quelle: Eigene Erhebung $(n=104)$ )

\begin{tabular}{|c|c|c|c|c|c|}
\hline \multicolumn{6}{|l|}{ Herkunft } \\
\hline Bundesland & Total & Prozent & Bundesland & Total & Prozent \\
\hline Niedersachsen & 22 & 21,2 & Baden-Württemberg & 3 & 2,9 \\
\hline Nordrhein-Westfalen & 18 & 17,3 & Brandenburg & 3 & 2,9 \\
\hline Sachsen & 17 & 16,3 & Berlin & 2 & 1,9 \\
\hline Sachsen-Anhalt & 8 & 7,7 & Rheinland-Pfalz & 2 & 1,9 \\
\hline Thüringen & 7 & 6,7 & Mecklenburg-Vorpommern & 2 & 1,9 \\
\hline Schleswig-Holstein & 5 & 4,8 & Bremen & 1 & 1,0 \\
\hline Hessen & 5 & 4,8 & Saarland & 1 & 1,0 \\
\hline Bayern & 4 & 3,8 & k. A. & 4 & 3,8 \\
\hline Altersstruktur & & & Geschlecht & & \\
\hline Alter & Total & Prozent & Geschlecht & Total & Prozent \\
\hline bis 35 Jahre & 24 & 23,1 & Männlich & 65 & 62,5 \\
\hline 36 bis 50 Jahre & 36 & 34,6 & Weiblich & 39 & 37,5 \\
\hline 51 bis 65 Jahre & 26 & 25,0 & & & \\
\hline 66 Jahre und älter & 17 & 16,3 & & & \\
\hline k. A. & 1 & 1,0 & & & \\
\hline
\end{tabular}

Als Startpunkt für die Untersuchung wurde das Plateau des Brocken gewählt, da es mehrere Dutzend Möglichkeiten für den Beginn einer Wanderung auf den Brocken hinauf gibt; damit wäre die Festlegung von Ausgabestandorten für die GPS-Logger erforderlich geworden. Nicht alle möglichen Orte für den Beginn einer Wanderung hätten jedoch mit Personal besetzt werden können. Eine beschränkte Auswahl bei den Ausgabeorten zum Erfassen der Wege auf den Brocken hätte die Routen stärker beeinflusst als im hier gewählten Vorgehen, da mit der Ansprache der Wanderer auf dem Brockenplateau sichergestellt werden konnte, dass alle Routen und Ziele beim Brockenabstieg einbezogen werden konnten.

Als Erstkontakt für die Ansprache der Probanden am Brocken wurde das Brockenplateau gewählt, was ein Gebiet von mehreren hundert Quadratmetern umfasst. Es boten sich dementsprechend unterschiedliche Standorte für die Gästeansprache an. Durch die Wahl des Befragungsortes sollte keine unbewusste Verfälschung der Ergebnisse erfolgen, wie z. B. durch die Ansprache von Wanderern in einer bestimmten Laufrichtung. Daher wurde bei der Auswahl der Befragungsstandorte darauf geachtet, dass die Ansprache nicht direkt am Beginn eines Weges stattfand. Des Weiteren wurde darauf Wert gelegt, dass der Erstkontakt mit den Wanderern im Freien erfolgte und nicht bei Anbietern gastronomischer oder sonstiger Dienstleistungen. Somit wurde verhindert, dass bestimmte Wandertypen (z. B. Selbstversorger) ausgeschlossen wurden. Eine Beeinflussung der Routenwahl ist nicht vollkommen auszuschließen, aber 99,1\% der Befragten gaben an, dass sie nicht beeinflusst wurden.

Die Rückgabe der GPS-Logger und die Durchführung der mündlichen Interviews konnten an jedem geäußerten Zielort erfolgen, um jede Wanderrichtung und jedes Wanderziel zuzulassen. Um den Personaleinsatz und die Fahrtkosten möglichst geringzuhalten, wurden jedoch Hauptbefragungsstandorte (Schierke Bahnhof und Nationalparkhaus, Parkplatz Torfhaus, Waldhotel Ilsenburg, Bad Harzburg/Seilbahn und Bad Harzburg/Tourist-Information) festgelegt. Für den Fall, dass Wanderer weitere Wanderziele anstrebten, die nicht durch Interviewer besetzt waren, wurden abendliche Termine in den Beherbergungsbetrieben vereinbart. ${ }^{9}$

Die Untersuchung fand an sechs Erhebungstagen vom 18. Mai bis 13. Juni 2013 statt. An den Untersuchungstagen konnten 129 GPS-Logger auf dem Brockenplateau an 334 Brocken-Wanderer ausgegeben werden. ${ }^{10}$ In sieben Fällen zeichneten die GPS-Logger nicht auf, bei drei Probanden nur teilweise und zwei Geräte wurden nicht zurückgegeben, sodass 117 vollständige Tracks vorhanden waren. Hiervon konnten mit fast allen Probanden im Anschluss auch Interviews geführt werden, sodass 104 formal vollständige Datensätze generiert werden konnten (= GPS-Tracks plus dazu gehörige Interviews).

Beim Lesen der folgenden Ergebnisse sollte bedacht werden, dass die Erhebung nur einen Zeitraum von knapp einem Monat umfasst. Hierdurch wird die Zusammensetzung der Probanden beeinflusst. Ebenso basieren die dargestellten Ergebnisse nur auf 104 ,kompletten“ GPS-Tracks. Dies hat Konsequenzen für die Aussagekraft der darauf beruhenden Überlegungen, da aufgrund der Stichprobenzahl

\footnotetext{
${ }^{9}$ Die Wanderer hatten außerdem die Möglichkeit, für den Fall, dass an ausgewählten Endpunkten kein Interviewer vor Ort verfügbar war, die GPS-Logger bei ausgewählten Betrieben abzugeben. Hiervon wurde in drei Fällen Gebrauch gemacht.

${ }^{10}$ Auch bei mehreren Personen wurde immer nur ein Gerät herausgegeben, da es für den Erhebungszweck keinen Sinn ergab, zwei, drei oder mehrere GPS-Geräte an ein Pärchen oder eine Wandergruppe auszugeben oder nur Wanderer einzubeziehen, die allein wanderten. Das Interview erfolgte zumeist gemeinsam mit dem/den Wanderpartner/n. Es wurden jedoch nur die Personen für das Interview ausgewählt, die sich zuvor dazu bereit erklärt hatten, die Fragen zu beantworten.
} 
Abb. 6 Hauptwanderströme der Brockenwanderer (Anzahl der Wandernden vom Brocken herunter). (Quelle: Eigene Darstellung $(n=104))$

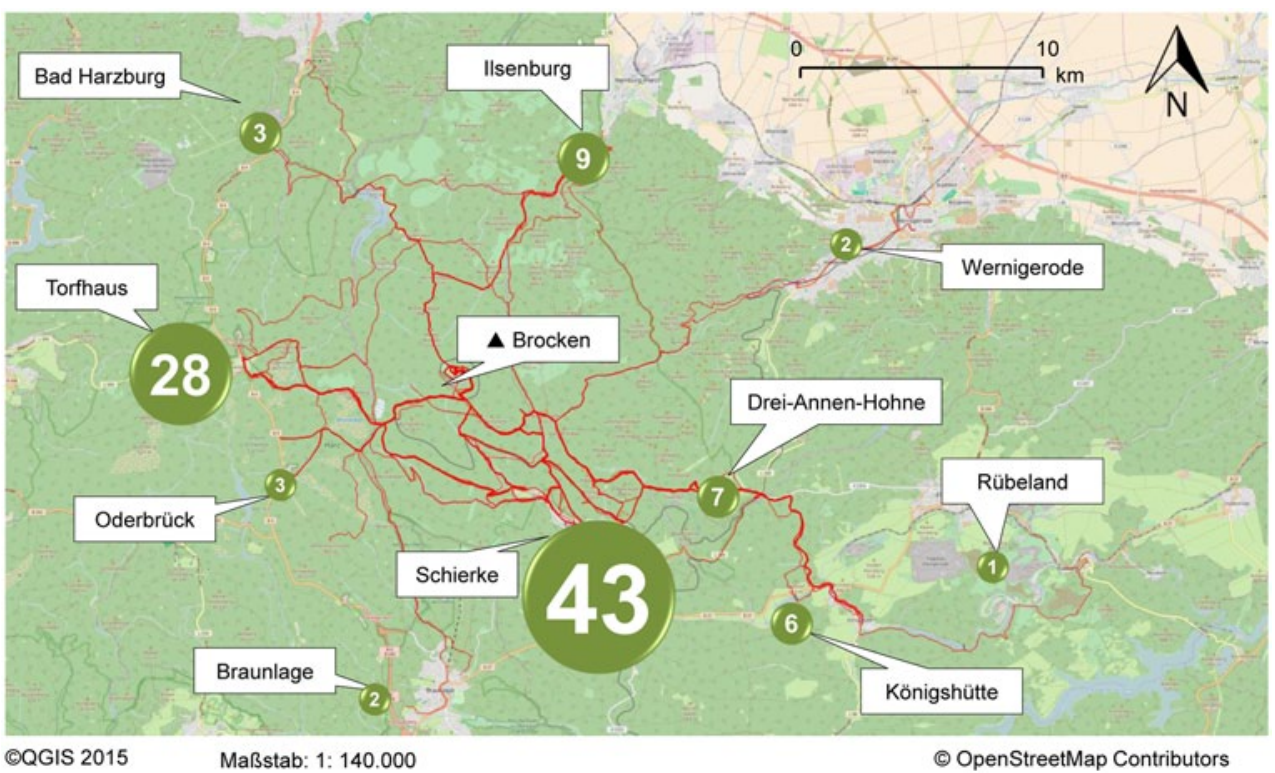

bereits kleine Ausreißer zu Ergebnisverzerrungen führen können (siehe mehr dazu unten). In diesem Zusammenhang sei jedoch erwähnt, dass die Erhebung am Brocken nur eine von mehreren Erhebungen im Laufe der Projektlaufzeit war. So wurden darüber hinaus in Wernigerode $(n=58)$, Goslar-Hahnenklee $(n=95)$ und im Zoo Hannover $(n=68)$ ähnliche Erhebungen durchgeführt. Für die hier bezweckte Darstellung der mit der Software-Anwendung verbundenen Möglichkeiten wurde mit der Brocken-Erhebung ein Fallbeispiel ausgewählt, das im ländlichen Raum situiert ist und sich somit von den meisten GPS-Tracking-Beiträgen abhebt, die sich zumeist eher auf städtische Gebiete beziehen.

\subsection{Beschreibung der Stichprobe}

Das Durchschnittsalter der befragten Teilnehmer betrug 47,7 Jahre. Mehr als die Hälfte der befragten Wanderer kam aus dem Harz sowie den angrenzenden Bundesländern. Tabelle 2 fasst diese und weitere soziodemographische Merkmale der Befragten zusammen.

\subsection{Ausgewählte Ergebnisse des GPS-Tracking}

Die meisten Wanderer waren vom Brockenplateau aus in Richtung Schierke $(41,3 \%)$ und Torfhaus $(26,9 \%)$ unterwegs. Eine Begründung dafür könnte darin liegen, dass beide Gemeinden die am nächsten gelegenen Zielpunkte für Wanderungen vom Brocken darstellen (die Entfernung nach Torfhaus beträgt ca. $8,5 \mathrm{~km}$ und nach Schierke je nach Route ca. 6 bis $8 \mathrm{~km}$ ). Hinzu kommt, dass der Brocken von Schierke aus mit der Harzer Schmalspurbahn (HSB) zu erreichen ist und einige Wanderer (14,2\% aller Befragten) die Kombination von Bahnfahrt (hinauf) und Wanderweg (hinab) nutzten. Diejenigen Wanderer, die Königshütte und
Rübeland als Ziel wählten, waren im Rahmen der Untersuchung Mehrtageswanderer, die dem Harzer-Hexen-Stieg folgten (vgl. Abb. 6).

\subsubsection{Differenzierung der Bewegungsströme}

Die Ergebnisse lassen sich nach bestimmten Eigenschaften, Bedürfnissen bzw. Verhaltensweisen der Probanden weiter differenzieren (Einbeziehung soziodemographischer (z. B. Alter, Geschlecht, Einkommen) und reiseverhaltensspezifischer Kriterien, wie Aufenthaltsart, Besuchshäufigkeit oder Wanderhäufigkeit). Derartige Kriterien werden in der touristischen Forschung und Praxis für die kundenbezogene Segmentierung herangezogen, um Zielgruppen besser erfassen und gezielter ansprechen zu können (vgl. Freyer 2011, S. 185). Auch in anderen Studien (Lau/McKercher 2006; McKercher/Lau 2008; McKercher/Shoval/Ng et al. 2012) wird eine derartige Vorgehensweise gewählt. Diese beziehen sich jedoch auf städtische Gebiete und nicht auf den ländlichen Raum, sodass sich die vorliegende Untersuchung in dieser Hinsicht abhebt.

So konnte unter anderem geprüft werden, ob es Unterschiede in den Bewegungsmustern von touristischen Besuchern in Abhängigkeit von der Besuchshäufigkeit im Harz gibt. Hierbei zeigte sich, dass es Unterschiede in der Länge, Dauer, Geschwindigkeit und vor allem der Wanderrichtung zwischen Erst-, Zweit-/Dritt- sowie Mehrfachbesuchern gab (vgl. Tab. 3 und Abb. 7). Exemplarisch sei erwähnt, dass die Erstbesucher ihre Wanderungen vor allem in Richtung Schierke durchführten - häufig auch verbunden mit einer Fahrt mit der HSB, um auf den Brocken zu gelangen. Die befragten Zweit- und Drittbesucher führten dagegen eine um mehr als $10 \%$ längere Wanderung als die beiden anderen Gruppen durch. 
Tab. 3 Wandereigenschaften von Touristen nach Besuchshäufigkeit. (Quelle: Eigene Erhebung $(n=94)$ )

\begin{tabular}{llll}
\hline & Erstbesucher $(n=27)$ & Zweit- und Drittbesucher $(n=25)$ & Mehrfachbesucher $(n=42)$ \\
\hline Ø Wanderlänge & $10,5 \mathrm{~km}$ & $11,9 \mathrm{~km}$ & $10,6 \mathrm{~km}$ \\
$\varnothing$ Wanderdauer & $03: 11: 15$ & $03: 33: 03$ & $03: 12: 45$ \\
$\varnothing$ Geschwindigkeit ohne Pausen & $3,7 \mathrm{~km} / \mathrm{h}$ & $3,6 \mathrm{~km} / \mathrm{h}$ & $3,8 \mathrm{~km} / \mathrm{h}$ \\
$\varnothing$ Geschwindigkeit inkl. Pausen & $3,4 \mathrm{~km} / \mathrm{h}$ & $3,4 \mathrm{~km} / \mathrm{h}$ & $3,4 \mathrm{~km} / \mathrm{h}$ \\
Wanderrichtung & $17 \times$ Schierke & $10 \times$ Schierke & $14 \times$ Torfhaus \\
& $4 \times$ Königshütte & $6 \times$ Torfhaus & $13 \times$ Schierke \\
& $3 \times$ Torfhaus & $2 \times$ Ilsenburg & $5 \times$ Ilsenburg \\
& $2 \times$ Ilsenburg & $2 \times$ Königshütte & $3 \times$ Bad Harzburg \\
& $1 \times$ Drei-Annen-Hohne & $2 \times$ Braunlage & $3 \times$ Drei-Annen-Hohne \\
& & $2 \times$ Drei-Annen-Hohne & $2 \times$ Wernigerode \\
& & $1 \times$ Rübeland & $2 \times$ Oderbrück \\
\hline
\end{tabular}

Es lässt sich feststellen, dass mit zunehmender HarzErfahrung vermehrt Wanderungen in weitere Richtungen (z. B. Torfhaus oder Ilsenburg) durchgeführt wurden. Dabei wurden nicht nur die Hauptrouten genutzt, sondern es wurde auch auf Nebenwegen gewandert. Die folgende Abbildung 7 zeigt die erhobenen Bewegungsmuster von „Erst-“, „Zweit- und Dritt-“" sowie „Mehrfachbesuchern“ (,Wiederholungsbesuchern“) im Vergleich.

\subsubsection{Analyse der Pol am Beispiel der Kategorie „, Rast- und Aussichtspunkte“"}

Insgesamt wurden im Rahmen der Untersuchung am Brocken 98 Pausen registriert, die sich der Pol-Kategorie „Rast- und Aussichtspunkte“"11 zuordnen lassen. In Abb. 8 sind alle unter dieser Pol-Kategorie registrierten Pausen grafisch dargestellt und nach Häufigkeit der Besuche durch Wanderer unterschiedlich groß gekennzeichnet.

Anhand der Übersicht zu den Handlungen am Pol in Tab. 4 fällt auf, dass ein Großteil der Pol in Verbindung mit Pausen (unter anderem Ausruhen, Genießen) und dem Verzehr mitgenommener Speisen bzw. Getränke aufgesucht wurden. Diese Aufenthalte zeichneten sich im Vergleich $\mathrm{zu}$ anderen Beweggründen durch eine etwas längere Verweildauer aus. Aber auch die Attraktivität der Natur- und Kulturlandschaft (Beispiel Kultur: Harzer Schmalspurbahn; Beispiel Natur: Aussicht) und das Fotografieren derselben waren Gründe, längere Stopps an den Pol einzulegen und dort zu verweilen. Bei der Tätigkeit „Unterhaltung“ fällt auf, dass es sich um einen Ausreißer handelt. Zwei von drei Personen hatten sich nach eigener Aussage bei schönem Wetter ,verquatscht“", sodass die durchschnittliche Verweildauer von der der anderen Aktivitäten stark abweicht.

\footnotetext{
${ }^{11}$ Die drei PoI-Kategorien „Verkehrsinfrastruktur“, „Sehenswürdigkeiten, Natur- und Kulturdenkmäler, Architektur“" und „Pausen/ Aussichten/Berggipfel“" wurden für diese Auswertung zu „Rast- und Aussichtspunkte“ zusammengefasst, da die einzelnen Kategorien zu geringe Fallzahlen für eine eigenständige Darstellung hatten.
}

Tab. 4 Aktivitäten der Wanderer an Rast- und Aussichtspunkten. (Sofern von einem Probanden zwei Handlungen gleichzeitig angegeben wurden, wurde die eine der einen Kategorie und die andere Aktion einer anderen Kategorie zugeordnet) (Quelle: Eigene Erhebung $(n=104)$; Mehrfachnennungen waren möglich)

\begin{tabular}{llll}
\hline Tätigkeitsbeschreibung & Anzahl & In Prozent & $\begin{array}{l}\text { Durchschnittliche } \\
\text { Verweildauer }\end{array}$ \\
\hline Rast/Pause allgemein & 39 & 35 & $15: 55$ \\
Gegessen/Getrunken & 30 & 27 & $17: 00$ \\
Fotografiert & 21 & 19 & $11: 14$ \\
Aussicht genossen & 19 & 17 & $09: 46$ \\
Dinge angeschaut & 11 & 10 & $11: 33$ \\
Informiert & 5 & 5 & $06: 24$ \\
Unterhaltung & 3 & 3 & $35: 40$ \\
Toilettenrast & 2 & 2 & $07: 30$ \\
Gewartet & 2 & 2 & $10: 30$ \\
Telefon/SMS & 2 & 2 & $08: 00$ \\
Hund & 2 & 2 & $11: 00$ \\
Jackenwechsel & 1 & 1 & $07: 00$ \\
Sonstiges & 6 & 5 & $07: 40$ \\
\hline
\end{tabular}

Zudem lassen sich differenzierte Aussagen zu den jeweiligen Aktionen an den einzelnen Wegepunkten treffen. Abbildung 9 visualisiert beispielsweise die Handlungen an den Rast- und Aussichtspunkten von vier der fünf am meisten besuchten Pol beim Brockenabstieg (der an 2 . Stelle liegende „Trudenstein“ ist nicht im Kartenausschnitt). Hieran wird ersichtlich, dass mehrere Wanderer auch Pausen entlang der Bahnschienen (siehe Punkte auf der Querlinie zwischen „Betriebsbahnhof Goetheweg“ und „Bahnübergang Abzweig Goetheweg") eingelegt haben. Für eine künftige Aufwertung der Wander-Infrastruktur könnten entlang dieser Wegestrecke weitere Bänke aufgestellt werden, da es hier aus Sicht der Gäste offenbar Interessantes zu sehen gibt.

Im Alltag und in der psychologischen Forschung wird Motivation als die Frage nach dem „Weshalb“ des menschlichen Verhaltens verstanden (vgl. Mey/Mruck 2010 und 
Abb. 7 Bewegungsmuster je nach Besuchshäufigkeit. (Quelle: Eigene Erhebung $(n=94)$ )

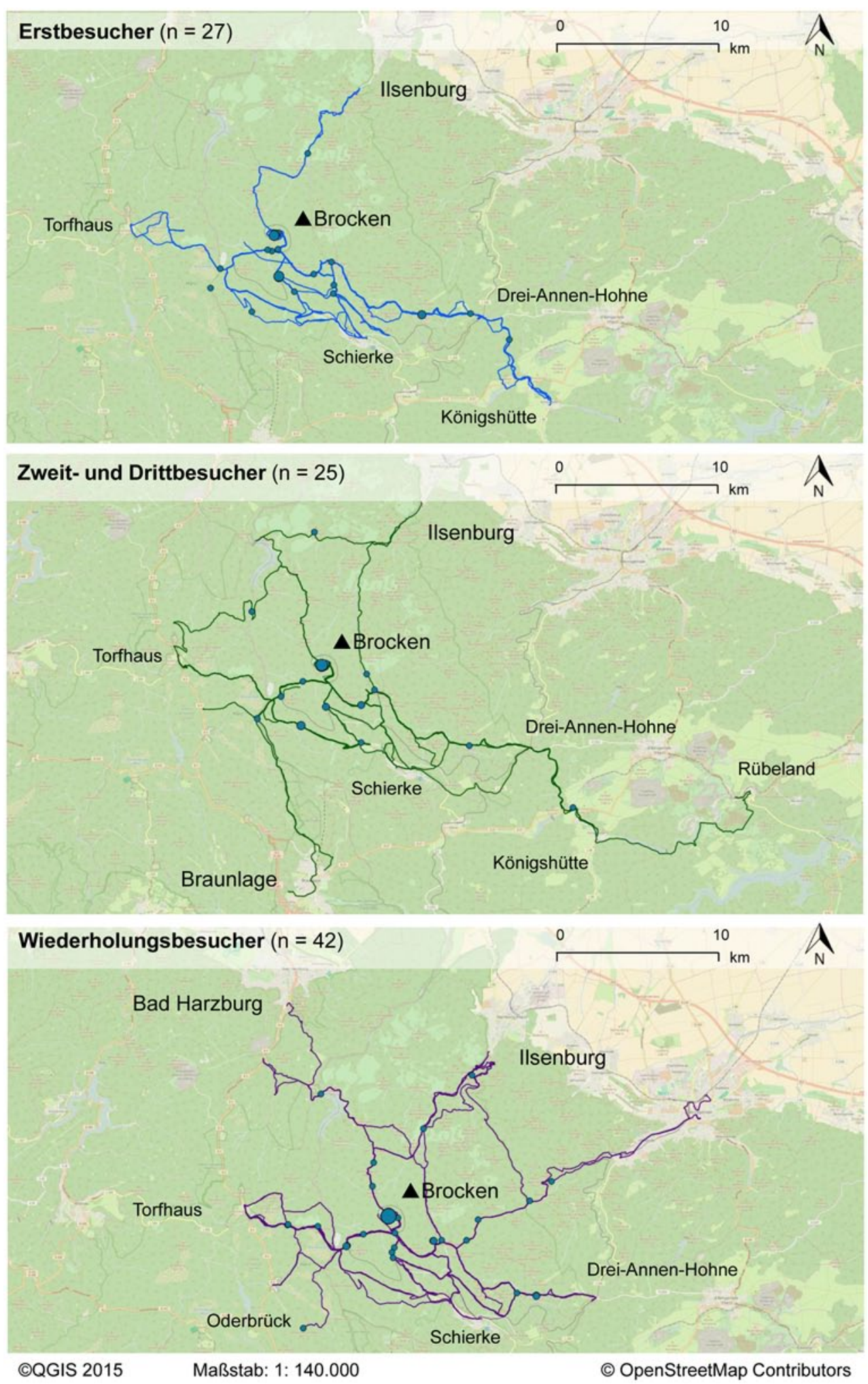

darin z. B. Straub/Chakkarath 2010, S. 198). Bei den Fragen zum Pol „Rast- und Aussichtspunkte“ geht es auch um das „Weshalb“, indem gefragt wurde: „Weshalb haben Sie an dieser Stelle länger verweilt bzw. was haben Sie speziell an dieser Stelle gemacht?“.
Für die Klassifikation der Angaben wurde die Bedürfnispyramide nach Maslow genutzt (vgl. Maslow 2002), wobei Angaben, die durch die Autoren nicht eindeutig 
Abb. 8 Besuchte Rast- und Aussichtspunkte. (Quelle: Eigene Erhebung $(n=104)$ (registrierte Pause $=$ mind. 3 min Aufenthalt am besuchten Pol))

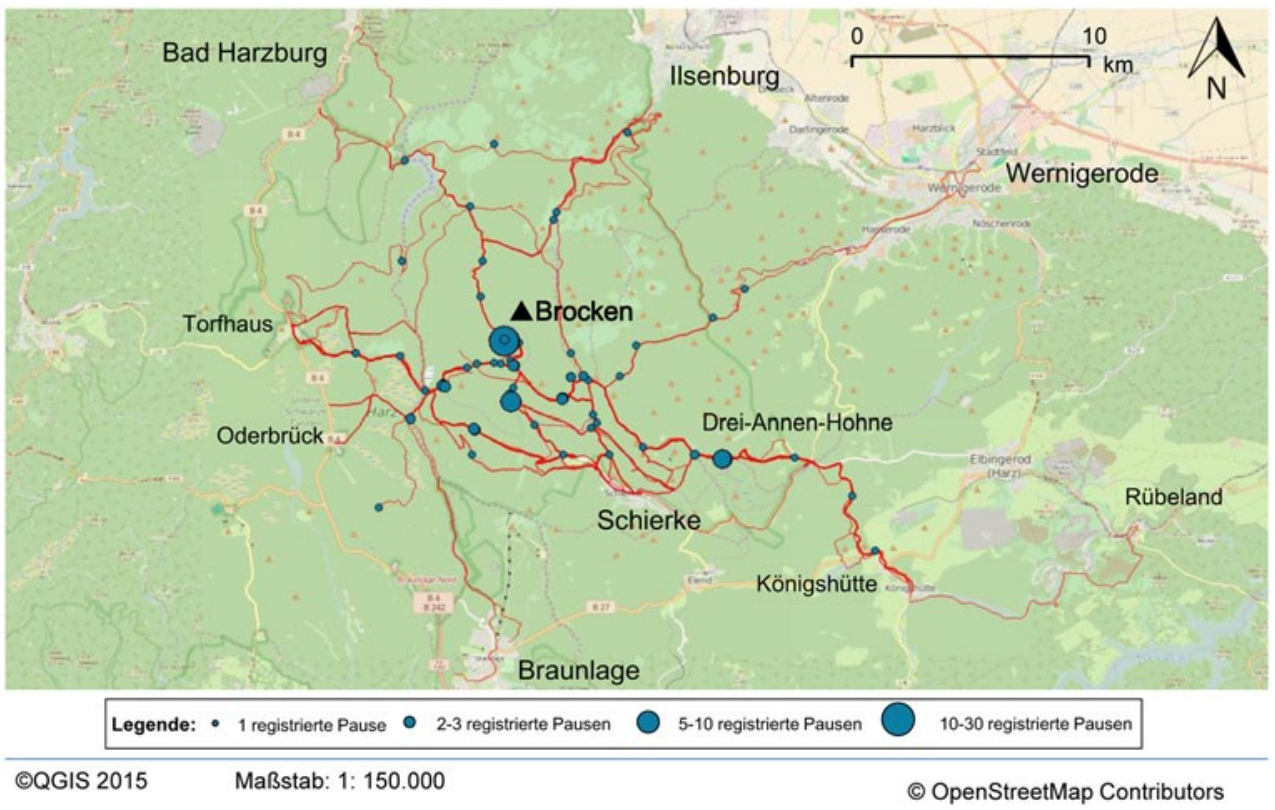

einer Bedürfnisebene zugeordnet werden konnten ${ }^{12}$, außer Betracht blieben. Die Maslow'sche Bedürnnishierarchie wird zu den Inhaltstheorien der Motivation gezählt. Inhaltsmodelle gehen der Frage nach, was und welche Faktoren Individuen motivieren; sie können in drei Gruppen unterschieden werden: monothematische, polythematische und athematische Theorien, wobei die meisten Motivtheorien polythematisch sind (vgl. Felser 2015, S. 101 f.). Auch wenn die Bedürfnispyramide von Maslow heute vielfach kritisiert wird, ist der Ansatz noch immer weit verbreitet. Die drei psychogenen Motive bei Maslow (also nicht die „biologischen“ Motive Hunger, Durst und Bedürfnis nach Sicherheit) decken sich über weite Strecken mit der ,(...) in der Psychologie vermutlich am weitesten verbreitete[n] polythematische[n] Taxonomie (...): Leistung, Macht und Anschluss (...)“(Felser 2015, S. 101).

Die meisten Antworten der Befragten ließen sich den Grundbedürfnissen (Essen, Trinken, Toilettenbesuch, Erholung) zuordnen, gefolgt von den Selbstverwirklichungsbedürfnissen. Soziale und Sicherheitsbedürfnisse waren seltener zu finden, was zwar einerseits im Widerspruch zur Dringlichkeitsabfolge der Maslow'schen Bedürfniskonzeption steht (Bedürfnisse der Selbstverwirklichung stehen auf der niedrigsten Dringlichkeitsstufe), andererseits schienen sich die Wanderer sicher zu fühlen (z. B. durch die vorhandene Wegweisung und den größtenteils gegebenen

\footnotetext{
${ }^{12}$ Fotografieren könnte beispielsweise eingeordnet werden als Zweck, der die Anerkennung durch Andere (= Wertschätzungsbedürfnisse) verfolgt, oder (als Handyfoto) zum Zwecke der Kommunikation (=Soziale Bedürfnisse) geschieht. Insgesamt 29 derartige Angaben konnten den Stufen der Bedürfnispyramide nicht einwandfrei zugeordnet werden $(21 \times$ Fotografieren, $1 \times$ Klettern und 7 Angaben zu aufgesuchten Zielen wie Museum, Zug oder Zugwechsel angeschaut).
}

Handyempfang rund um den Brocken) und trachteten eher danach, ein oder mehrere Selbstverwirklichungsbedürfnisse zu befriedigen als ihre sozialen Bedürfnisse (vgl. Abb. 10).

Diese Ergebnisse sind für Tourismusorganisationen, Wandervereine oder Nationalparke von Interesse, die Gebiete verwalten, die eine ähnliche Publikumsstruktur wie der Harz haben und z. B. ein Wanderwegekonzept entwickeln oder ihre vorhandenen Wanderwege aufwerten möchten. Kurz gesagt müssen zunächst die Grund- und Sicherheitsbedürfnisse befriedigt werden, unter anderem durch Rastmöglichkeiten, Toiletten und ein Beschilderungskonzept. Um aber ein qualitativ hochwertiges wandertouristisches Produkt zu schaffen und ein Alleinstellungsmerkmal herzustellen, müssen auch die anderen Bedürfnisse erfüllt werden. Wertschöpfungs- und Entwicklungsbedürfnisse lassen sich beispielsweise durch neu geschaffene Aussichts- und Fotopunkte befriedigen, die gezielt angelegt werden.

An den Pol kann durch entsprechende Fragen auch die Zufriedenheit mit einzelnen Anbietern analysiert werden. In Gästebefragungen auf Destinationsebene (vor allem Orts-, Regional- oder Bundeslandebene) werden fast immer auch Leistungsbestandteile des touristischen Angebotes (z. B. Preis-Leistungs-Verhältnis, Ortsbild, Qualität der Gastronomie) bewertet (vgl. Freyer/Groß 2006, S. 105 ff.). Solche Bewertungen werden jedoch für die gesamte Destination erfasst und nicht spezifiziert für einen einzelnen Betrieb. Außerdem werden sie selten am Tag der Inanspruchnahme bzw. des Erlebens durchgeführt, sondern meist erst einige Tage später, sodass andere Erlebnisse den eigentlichen Eindruck überformen können. Touristische Unternehmen können mithilfe eigener Erhebungen zwar auch eine Befragung ihrer Gäste durchführen, dann wiederum ist eine Vergleichbarkeit zwischen den einzelnen Betrieben 
Abb. 9 Ausgewählte Rast- und Aussichtspunkte beim Brockenabstieg. (Quelle: Eigene Darstellung; Mehrfachnennungen waren möglich)

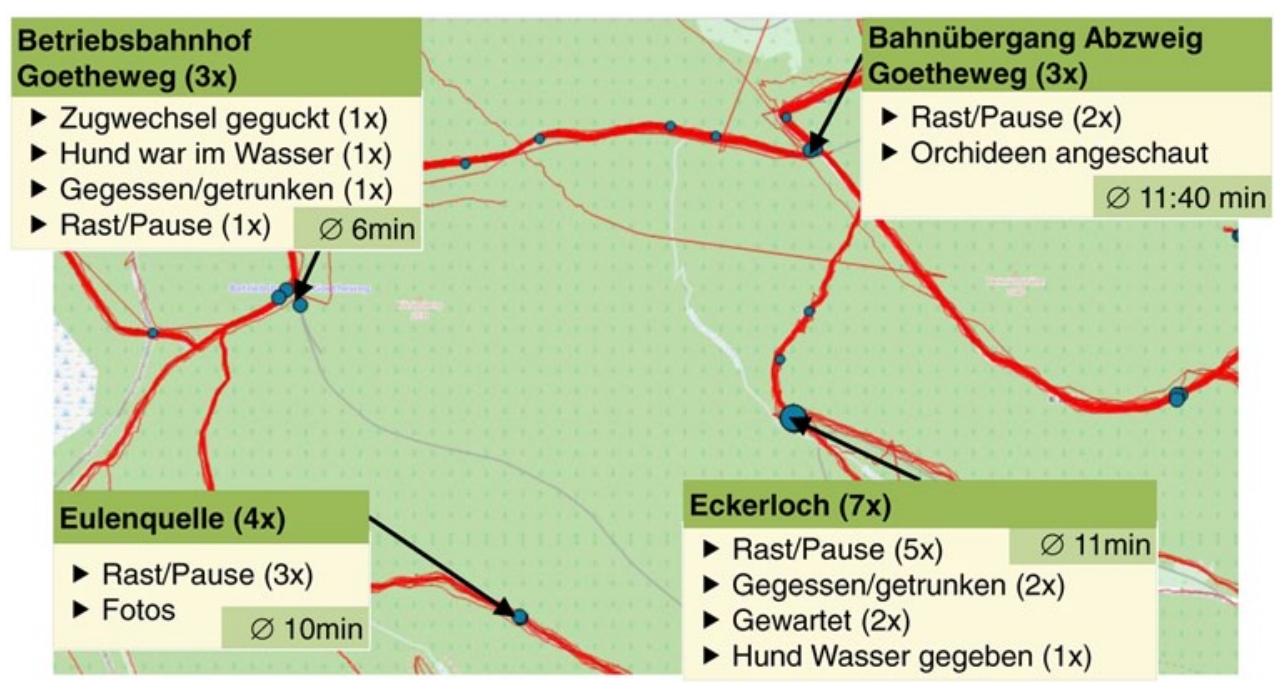

\begin{tabular}{|c|c|c|c|}
\hline Bedürfnisebene & Allgemeine Erläuterung & $\begin{array}{c}\text { Beispiele in der } \\
\text { Befragung }\end{array}$ & $\begin{array}{l}\text { Anzahl der } \\
\text { Nennungen }\end{array}$ \\
\hline \multirow[t]{2}{*}{$\begin{array}{l}\text { Selbstverwirk- } \\
\text { lichung }\end{array}$} & $\begin{array}{r}\text { Bringt zum Ausdruck, dass der Mensch } \\
\text { das sein will, was er sein kann, } \\
\text { z.B. Unabhängigkeit, Freude, Glück, } \\
\text { machen, wozu er fähig ist }\end{array}$ & $\begin{array}{l}\text { Aussicht genießen, } \\
\text { Orchideen anschauen }\end{array}$ & 25 \\
\hline & $\begin{array}{r}\text { Verlangen nach Anerkennung durch } \\
\text { persönliche Umwelt, } \\
\text { z.B. soziales Ansehen, Macht, } \\
\text { Beachtung, Lob, Anerkennung, } \\
\text { Prestige, Freiheit }\end{array}$ & $\begin{array}{l}\text { Fotografieren (im Sinne } \\
\text { von Anerkennung durch } \\
\text { Andere), Klettern (im } \\
\text { Sinne von Freiheit } \\
\text { erleben) }\end{array}$ & 0 \\
\hline $\begin{array}{l}\text { Soziale } \\
\text { Bedürfnisse }\end{array}$ & $\begin{array}{r}\text { Wunsch nach Geborgenheit in } \\
\text { der menschlichen Umwelt, } \\
\text { z.B. Liebe, Freundschaft, Kontakt, } \\
\text { Kommunikation, } \\
\text { Zusammengehörigkeitsgefühl }\end{array}$ & $\begin{array}{l}\text { Kommunikation, SMS } \\
\text { schreiben, Telefonieren, } \\
\text { Motivation der Mit- } \\
\text { Teilnehmer }\end{array}$ & 8 \\
\hline $\begin{array}{l}\text { Sicherheits- } \\
\text { bedürfnisse }\end{array}$ & $\begin{array}{r}\text { Beziehen sich auf den } \\
\text { Schutz vor möglichen } \\
\text { Bedrohungen/Gefahren, } \\
\text { z.B. Schutz bei Krankheit/Unfall, } \\
\text { Vorsorge für die Zukunft }\end{array}$ & $\begin{array}{l}\text { Information, nach Weg } \\
\text { fragen, Orientierung }\end{array}$ & 9 \\
\hline Grundbedürfnisse & $\begin{array}{r}\text { Haben körperliche Grundlage, } \\
\text { ihre Befriedigung ist Voraus- } \\
\text { setzung für Lebenserhaltung, } \\
\text { z.B. Essen, Trinken, Schlafen, } \\
\text { Wohnen, Sexualität }\end{array}$ & $\begin{array}{l}\text { Essen, Trinken, } \\
\text { Toilettenrast, } \\
\text { Pause/Rast als } \\
\text { Erholung }\end{array}$ & 78 \\
\hline
\end{tabular}

Abb. 10 Gründe für den Stopp am Pol „Rast- und Aussichtspunkte“. (Quelle: in Anlehnung an Freyer 2015, S. 79)

meist nicht gegeben, da die Betriebe ihre Bewertungen nicht öffentlich machen (wollen) und die Befragungen an sich auch nicht in dem Maße vergleichbar sind, wie es bei dem hier vorgestellten Erhebungsinstrumentarium der Fall ist. Darüber hinaus sei hier erwähnt, dass die Applikation die tatsächlich besuchten Pol automatisch anzeigt und Proband und Interviewer durch Rücksprache den genauen Betrieb bestimmen können. Selbst bei ortsunkundigen Probanden kann so anhand der Karte auf dem Tablet-PC der von ihnen besuchte Betrieb ermittelt werden.

Exemplarisch wird nachfolgend die Zufriedenheit mit dem gastronomischen Angebot derjenigen dargestellt, die einen gastronomischen Betrieb aufgesucht haben. Die Bewertungen sind in anonymisierter Form Tabelle 5 zu entnehmen. Hierbei wird ersichtlich, dass die Probanden die Speisen bzw. Getränke der gastronomischen Einrichtungen auf dem Brocken selbst etwas besser bewerteten als die der 
Tab. 5 Bewertung der Zufriedenheit mit dem gastronomischen Angebot

\begin{tabular}{|c|c|c|c|c|c|c|}
\hline & \multicolumn{2}{|c|}{$\begin{array}{l}\text { Gastronomische Einrichtungen auf } \\
\text { dem Brocken }\end{array}$} & \multicolumn{2}{|c|}{$\begin{array}{l}\text { Gastronomische Einrich-tungen } \\
\text { rund um den Bro-cken bzw. in den } \\
\text { Zielorten }\end{array}$} & \multicolumn{2}{|c|}{ Gastronomie gesamt } \\
\hline & Mittelwert & Standard-abweichung & Mittelwert & Standard-abweichung & Mittelwert & Standard-abweichung \\
\hline Anzahl Besuche & 10 & - & 17 & - & 27 & - \\
\hline$\varnothing$-Ausgabe & $7,40 €$ & $4,70 €$ & $6,80 €$ & $5,65 €$ & $7,04 €$ & $5,15 €$ \\
\hline \multicolumn{7}{|c|}{ Zufriedenheitsbewertung 1 = „sehr zufrieden“; 5 = „unzufrieden“" } \\
\hline $\begin{array}{l}\text { Ambiente, } \\
\text { Gesamteindruck }\end{array}$ & 2,56 & 0,73 & 2,14 & 0,54 & 2,30 & 0,64 \\
\hline Servicequalität & 2,38 & 0,52 & 2,23 & 0,60 & 2,29 & 0,56 \\
\hline Freundlichkeit & 2,00 & 0,76 & 1,92 & 0,64 & 1,95 & 0,67 \\
\hline Speisen, Getränke & 1,89 & 0,60 & 2,00 & 0,68 & 1,96 & 0,64 \\
\hline Sauberkeit & 1,78 & 0,44 & 1,57 & 0,51 & 1,65 & 0,49 \\
\hline Gesamtzufriedenheit & 2,33 & 0,87 & 1,86 & 0,36 & 2,04 & 0,64 \\
\hline
\end{tabular}

anderen gastronomischen Einrichtungen der Umgebung. Das Ambiente, die Servicequalität, Sauberkeit und Freundlichkeit sowie die Gesamtzufriedenheit wurden jedoch bei den Einrichtungen rund um den Brocken bzw. in den Zielorten für etwas besser befunden.

In einem Workshop mit einem Dutzend Projektpartner aus der Region (z. B. Landeswanderverband, Hoteliers, Tourismusorganisationen, Nationalparkverwaltung, Ministerium für Wirtschaft und Wissenschaft Sachsen-Anhalt) wurden ausgewählte Ergebnisse der Untersuchung am Brocken präsentiert. Dabei wurde ein ausgesprochen positives Feedback zu den Erkenntnissen gegeben und weitere Schritte wurden verabredet. So sollen die Erkenntnisse beispielsweise in der Nationalparkverwaltung und beim Landeswanderverband genutzt und auch an die Wegewarte des Harzklubs (zuständig für die Pflege und Wartung der Wanderwege im Harz) weitergegeben werden. Die genannten Institutionen haben zugesichert, dass sie mit den Ergebnissen gezielt Schwachstellen auf den Wanderwegen rund um den Brocken beheben bzw. bei neuen Planungen die Ergebnisse einbeziehen möchten. Als ein Beispiel kann hier die Nutzung der erhobenen Daten für die Überarbeitung des Nationalpark-Wegeplans der Nationalparkverwaltung Harz gelten.

\section{Fazit}

Bei der Darstellung der verschiedenen Methoden der Mobilitätserfassung wird deutlich, dass die Erfassung personenbezogener Mobilität auf einer Ebene, die Individuum, Handlung (im Sinne von Aktivität am Polnt of Interest (PoI)) und räumliche Mobilität vereint, bis heute durch eine fehlende Ortsgenauigkeit limitiert wird. Dieser methodische Mangel kann durch die vorgestellte Android-Applikation und die Verknüpfung von sich ergänzenden empirischen Techniken (GPS-Tracking und Face-to-Face-Befragung direkt im Anschluss an die zurückgelegten Wege) über- wunden werden. Daraus eröffnet sich ein aussichtsreicher Ansatz für die Erforschung des raum-zeitlichen Verhaltens von Personen (z. B. Wanderern, Besuchern von Freizeiteinrichtungen).

In Bezug auf die bereits genannten Limitierungen sollen abschließend einige Verbesserungsvorschläge gemacht und Schlussfolgerungen für weitere Untersuchungen gezogen werden. Eine stärkere Fokussierung auf VorOrt-Entscheidungen und die diesen zugrunde liegenden Motivationen ist prinzipiell denkbar und mit geringem Aufwand umsetzbar. Hierfür müssten die Fragestellungen überarbeitet werden.

Vorteile der dargestellten Anwendung sind, a) dass sie es ermöglicht, vergleichsweise kostengünstig eine höhere Stichprobe von Bewegungsmustern zu generieren, b) dass sich durch die weitgehende Automatisierung der Datenerfassung und -verarbeitung Prozesse vereinfachen lassen, die ansonsten häufig manuell $\mathrm{zu}$ bewerkstelligen sind, und c) dass die Implementierung einer Vielzahl von Befragungen mit unterschiedlichen Ergebnissen möglich ist. Beispielsweise können alle Wegepunkte einzeln nach Merkmalen wie Aufenthaltsdauer, Aktivität, Zufriedenheit und Verbesserungsvorschlägen betrachtet und zusätzliche Differenzierungen (z. B. nach soziodemographischen oder verhaltensspezifischen Merkmalen) vorgenommen werden. Auch der Anlass, weshalb sich die Probanden an den jeweiligen Pol aufgehalten haben, oder ortsgenaue Entscheidungen können so zeitnah erfasst werden.

Ein weiterer Vorteil der neu entwickelten Anwendung besteht darin, dass die zurückgelegten Strecken der Probanden direkt vor Ort am Tablet-Computer ausgelesen und ausgewertet werden können. Mit dem Ziel, ortsgenaue Ergebnisse zu den Aufenthaltsorten der Probanden zu ermitteln, werden mithilfe dieser App alle Punkte aufgezeigt, an denen sich die Testpersonen beispielsweise länger als drei Minuten aufhielten - der bei anderen Methoden (z. B. Mobilitätstagebücher) geäußerten Kritik, dass sich die Probanden nicht immer an alle wichtigen Momente erinnern (vgl. Groß 
2005, S. 153; Groß/Groß/Freyer 2006, S. 50), kann mit der Aufzeichnung aller Stopps und der automatischen Anzeige begegnet werden. Über die in ersten Projekten erhobenen Daten hinaus lassen sich die Fragen mit geringem Aufwand erweitern bzw. anpassen, ${ }^{13}$ um z. B. auch Daten zu lebensstilbezogenen Merkmalen, Präferenzen bei den Wegen (z. B. gewünschter Untergrund) und Zeitzwängen (vgl. Millonig/ Gartner 2008, S. 1 f.) zu erfassen oder mehr als die bisher fünf erfassten Pol zu analysieren.

Sowohl die mithilfe der Face-to-Face-Befragungen zu erhebenden Informationen als auch die erstellten Karten mit den Gästeströmen können - ähnlich den Laufwegeanalysen oder dem „Shopper-Tracking“ im Groß- und Einzelhandel $^{14}$ - für Entscheidungsträger in Stadtverwaltungen und Tourismusorganisationen hilfreich sein. Mithilfe der darstellbaren Bewegungsmuster können Überlegungen hinsichtlich der Platzierung von Bänken, Papierkörben, Wegweisern und Informationstafeln, Schutzhütten o. ä. gestützt und begründet werden, die vorhandene (touristische) Beschilderung verbessert werden oder überprüft werden, ob sensible Gebiete (z. B. im Nationalpark) aufgesucht werden. Darüber hinaus kann beispielsweise bei Untersuchungen in städtischen Räumen der Frage nachgegangen werden, wo z. B. touristisch relevante Einrichtungen bzw. Geschäfte eröffnet werden sollten, da erfasst werden kann, welche Orte eine höhere Frequentierung haben. Es kann auch ermittelt werden, welche Pol die Besucher vermehrt ansteuern, also könnte eine Analyse der Anziehungskraft eines Aufmerksamkeitspunkts erfolgen, welches die „Hot Spots“ sind und welche Pol demgegenüber weniger bekannt sind. Eine Besucherlenkung könnte ebenfalls hieran ansetzen.

Mithilfe der jeweiligen Aufenthaltsdauer kann darüber hinaus eruiert werden, welche Pol nur kurz besucht werden, da sich an dieser Stelle z. B. keine Bänke befinden oder die Sonne einfällt, und wo die Besucher dagegen gerne länger verweilen. Diese Informationen können dabei helfen, das touristische Angebot besser zu vermarkten, indem beispielsweise unterschiedliche Pauschalprogramme oder Informationsbroschüren für Erst- und Mehrfachbesucher aufgelegt werden oder Lösungsansätze gefunden werden, wie sich Gäste an einem bestimmten Ort länger aufhalten können.

\footnotetext{
${ }^{13}$ Der Inhalt der Fragen kann durch eine XML-Konfigurationsdatei definiert werden, wobei sechs vordefinierte Fragetypen zur Verfügung stehen: 1) geschlossene, dichotome Frage mit Einfachauswahl ,ja/nein“, 2) geschlossene Frage (Einfachauswahl mit mehreren Antwortvorgaben), 3) geschlossene Hybridfrage (Einfachauswahl mit optionalem Textfeld), 4) geschlossene Hybridfrage (Mehrfachauswahl mit optionalem Textfeld), 5) Bewertungsfragen (Einfachauswahl je Bewertungskriterium), 6) offene Frage.

${ }^{14}$ Weiterführende Informationen zu derartigen Untersuchungen finden sich beispielsweise bei Kilzer 2009, Sorensen 2009 und Hunstiger 2011.
}

\section{Literatur}

Alvares, L. O.; Bogorny, V.; Kuipers, B.; de Macedo, J. A. F.; Moelans, B.; Vaisman, A. (2007): A model for enriching trajectories with semantic geographical information. In: Proceedings of the 15th annual ACM international symposium on advances in geographic information systems. Seattle, Washington. https://doclib.uhasselt. be/dspace/bitstream/1942/7905/1/07-GIS.pdf (13.07.2015).

Arrowsmith, C.; Chhetri, P. (2003): Port Campbell National Park Patterns of Use. A report for the development of visitor typology as input to a generic model of visitor movements and patterns of use. Melbourne.

Bachu, P. K.; Dudala, T.; Kothuri, S. M. (2001): Prompted Recall in Global Positioning System Survey: Proof-of-Concept Study. In: Transportation Research Record 1768, 106-113.

Bauder, M. (2011): GPS-Erfassung und GIS-Analyse individueller Personenmobilität. (Zugleich Master Thesis, Albert-LudwigsUniversität Freiburg). http://www.freidok.uni-freiburg.de/volltexte/8385/ (10.12.2013).

Bauder, M. (2012): Erfahrungen aus dem GPS-Tracking individueller Mobilität von Touristen - Konsequenzen für die Weiterentwicklung als neue humangeographische Methode. In: Strobl, J.; Blaschke, T.; Griesebner, G. (Hrsg.): Angewandte Geoinformatik. Berlin, Offenbach, 420-429.

Bieger, T. (2008): Management von Destinationen. München, Wien.

Büscher, M.; Urry, J. (2009): Mobile Methods and the Empirical. In: European Journal of Social Theory 12, 1, 99-116.

Canzler, W.; Knie, A. (1998): Möglichkeitsräume: Grundrisse einer modernen Mobilitäts- und Verkehrspolitik. Wien.

Carlstein, T.; Parkes, D. N.; Thrift, N. (1978): Timing Space and Spacing Time. London.

Cerwenka, P. (1999): Mobilität und Verkehr: Duett oder Duell von Begriffen?. In: Der Nahverkehr 17, 05, 34-37.

Cherem, G. J.; Driver, B. L. (1983): Visitor Employed Photography: A Technique to Measure Common Perceptions of Natural Environments. In: Journal of Leisure Research 15, 1, 65-83.

Clifford, E.; Zhang, J.; Stopher, P. (2008): Determining trip information using GPS data. Sydney. = Working Paper ITLS-WP-08-01.

Cohen, S. A.; Duncan, T.; Thulemark M. (2015): Liftstyle mobilities: The crossroads of travel, leisure and migration. In: Mobilities 10, $1,155-172$.

Czaplicki, A. (2009): Nutzung von GPS-Daten - Analyse der Besucherwege des Leipziger Zoos. In: König, C.; Stahl, M.; Wiegand, E. (Hrsg.): Nicht-reaktive Erhebungsverfahren. Bonn, 73-81.

Diewitz, U.; Klippel, P.; Verron, H. (1998): Der Verkehr droht die Mobilität zu ersticken. In: Internationales Verkehrswesen 50, 3, 72-74.

Downs, R.; Stea, D. (1982): Kognitive Karten - Die Welt in unseren Köpfen. New York.

Edwards, D.; Griffin, T. (2013): Understanding tourists' spatial behavior: GPS tracking as an aid to sustainable destination management. In: Journal of Sustainable Tourism 21, 4, 580-595.

Edwards, D.; Griffin, T.; Hayllar, B.; Dickson, T.; Schweinsberg, S. (2009): Understanding Tourist ,Experiences' and ,Behaviour' in Cities - An Australian Case Study. Queensland.

Felser, G. (2015): Werbe- und Konsumentenpsychologie. Heidelberg.

Fennell, D. A. (1996): A tourist space-time budget in the Shetland Islands. In: Annals of Tourism Research 23, 4, 811-829.

Franz, P. (1984): Soziologie der räumlichen Mobilität. Eine Einführung. Frankfurt am Main.

Freyer, W. (2011): Tourismus-Marketing - Marktorientiertes Management im Mikro- und Makrobereich der Tourismuswirtschaft. München.

Freyer, W. (2015): Tourismus - Einführung in die Fremdenverkehrsökonomie. Berlin, München, Boston.

Freyer, W.; Groß, S. (2006): Gästebefragungen in der touristischen Marktforschung - Leitfaden für die Praxis. Dresden. 
Graaff, N. (2014): VEP \& GPS als Methode zur Untersuchung natürlicher Aufmerksamkeitspunkte in Nationalparken. Poster zur 18. Jahrestagung der Deutschen Gesellschaft für Tourismuswissenschaft vom 13.-15.11.2014. Köln.

Groß, M. S.; Groß, S.; Freyer, W. (2006): Mobilitätsverhalten im Tourismus - Methodenstudie zur Erfassung des Mobilitätsverhaltens von Touristen am Aufenthaltsort. In: Zeitschrift für Verkehrswissenschaft 77, 1, 29-55.

Groß, S. (2005): Mobilitätsmanagement im Tourismus. Dresden.

Hägerstrand, T. (1970): What about people in Social Sciences? In: Papers of the Regional Science Association 24, 1, 7-21.

Hartmann, R. (1988): Combining field methods in tourism research. In: Annals of Tourism Research 15, 1, 88-105.

Hautzinger, H.; Pfeiffer, M.; Tassaux-Becker, B. (1994): Mobilität: Ursachen, Meinungen, Gestaltbarkeit. Heilbronn.

Higham, E. C.; Holt, A.; Kearsley, C. (1996): Tourist flow reasoning - the spatial similarities of tourist movements. In: Proceedings for the Spatial Information Research Centre's 8th Colloquium, University of Otago. Dunedin, 69-78.

Hunstiger, G. (2011): Dem Shopper auf der Spur: praxiserprobte Marktforschungsmethoden. In: Frey, U. R.; Hunstiger, G.; Dräger, P. (Hrsg.): Shopper-Marketing - Mit Shopper Insights zu effektiver Markenführung bis an den POS. Wiesbaden, 99-130.

IDC - International Data Corporation (2015): Smartphone OS Market Share. http://www.idc.com/prodserv/smartphone-os-market-share. jsp (30.10.2015).

Inskeep, E. (1991): Tourism Planning: An Integrated and Sustainable Development Approach. New York.

inspektour $\mathrm{GmbH}$; ift $\mathrm{GmbH}$ - ift Freizeit- und Tourismusberatung GmbH (2012): Permanente Gästebefragung Sachsen-Anhalt 2011/12 - Berichtsband mit Endergebnissen. Köln, Hamburg, Potsdam.

Institut für Demoskopie (1956): Nato-Wanderausstellung 1956. Ergebnisse einer Umfrage unter Ausstellungsbesuchern (IfD-Umfrage 186). Allensbach am Bodensee. $=$ IfD-Bericht 531.

Job, H.; Woltering, M.; Schamel, J.; Merlin, C. (2014): Regionalökonomische Effekte des Nationalparks Harz. Würzburg.

Kaiser, F. G. (1993): Mobilität als Wohnproblem - Ortsbindung im Licht emotionaler Regulation. Bern.

Kazig, R.; Popp, M. (2011): Unterwegs in fremden Umgebungen - Ein praxeologischer Zugang zum ,wayfinding“ von Fußgängern. In: Raumforschung und Raumordnung 69, 3, 3-15.

Keul, A. G.; Kühberger, A. (1996): Die Straße der Ameisen - Beobachtungen und Interviews zum Salzburger Städtetourismus. München, Wien.

Kilzer, F. (2009): Verhaltensbeobachtungen am Point-of-Sale (POS). In: König, C.; Stahl, M.; Wiegand, E. (Hrsg.): Nicht-reaktive Erhebungsverfahren. Bonn, 33-43.

Kleehaupt, B. (1997): Verkehrliche Mobilitätskritik aus ökonomischer Sicht. Frankfurt am Main.

Lau, G.; McKercher, B. (2006): Understanding Tourist Movement Patterns in a Destination: A GIS Approach. In: Tourism and Hospitality Research 7, 1, 39-49.

Lopez, C. (2014): Faszination Mobile - Verbreitung, Nutzungsmuster und Trends. Bundesverband Digitale Wirtschaft in Kooperation mit Google und TNS Infratest. Düsseldorf.

Maslow, A. H. (2002): Motivation und Persönlichkeit. Reinbek.

McKercher, B.; Lau, G. (2008): Movement Patterns of Tourists within a Destination. In: Tourism Geographies 10, 3, 355-374.

McKercher, B.; Shoval, N.; Ng, E.; Birenboim, A. (2012): First and Repeat Visitor Behaviour: GPS Tracking and GIS Analysis in Hong Kong. In: Tourism Geographies 14, 1, 147-161.

Mey, G.; Mruck, K. (2010) (Hrsg.): Handbuch Qualitative Forschung in der Psychologie. Wiesbaden.

Millonig, A.; Gartner, G. (2007): Monitoring Pedestrian Spatio-Temporal Behavior. In: Gottfried, B. (Hrsg.): BMI - Behaviour Monitoring and Interpretation. Proceedings of the Workshop on Behaviour Monitoring and Interpretation - BMI 2007. Osnabrück, 29-42.
Millonig, A.; Gartner, G. (2008): Shadowing-Tracking - Interviewing: How to Explore Human Spatio-Temporal Behaviour Patterns. In: Gottfried, B.; Aghajan, H. (Hrsg.): Workshop on Behaviour Monitoring and Interpretation - BMI 2008 in Conjunction with 31 st German Conference on Artificial Intelligence. Bremen, 1-14. $=$ TZI-Bericht, 48 .

Murphy, P. E. (1992): Urban tourism and visitor behavior. In: American Behavioral Scientist 36, 2, 200-211.

Neis, P. (2008): Location Based Services mit OpenStreetMap Daten. Masterarbeit. Mainz.

Nielsen, N. C.; Blichfeldt, B. S. (2009): Where do they go? Monitoring Tourist Mobility at the Destination. In: 18th Nordic Symposium in Tourism and Hospitality Research. 22nd - 25th October 2009, Esbjerg, Dänemark.

OSM - Open Street Map (2015a): Stats. http://wiki.openstreetmap. org/wiki/Stats (04.11.2015).

OSM - Open Street Map (2015b): Map Features. http://wiki. openstreetmap.org/wiki/Map Features (12.10.2015).

Ramm, F.; Topf, J. (2010): OpenStreetMap - Die freie Weltkarte nutzen und mitgestalten. Berlin.

Sheller, M.; Urry, J. (2006): The new mobilities paradigm. In: Environment and Planning A 38, 2, 207-226.

Shoval, N.; Isaacson, M. (2010): Tourist Mobility and Advanced Tracking Technologies. New York.

Sorensen, H. (2009): Inside the mind of the shopper: The science of retailing. Upper Saddle River.

Spangenberg, T. (2014): Development of a Mobile Toolkit to Support Research on Human Mobility Behavior Using GPS Trajectories. In: Information Technology \& Tourism (Special Issue „ICT for Sustainable Tourism“) 14, 4, 317-346.

Statistisches Bundesamt (2007): Gliederung der Klassifikation der Wirtschaftszweige, Ausgabe 2008 (WZ 2008). Wiesbaden.

Statistisches Bundesamt (2015): Binnenhandel, Gastgewerbe, Tourismus - Ergebnisse der Monatsergebnisse im Tourismus, Dezember 2014. Wiesbaden.

Stopher, P.; Prasad, C.; Zhang, J. (2010): Comparing GPS and prompted recall data records. Sydney. $=$ Working Paper ITLS-WP-10-24

Straub, J.; Chakkarath, P. (2010): Kulturpsychologie. In: Mey, G.; Mruck, K. (Hrsg.): Handbuch Qualitative Forschung in der Psychologie. Wiesbaden, 195-209.

Thornton, P. R.; Williams, A. M.; Shaw, G. (1997): Revisiting time-space diaries - An exploratory case study of tourist behavior in Cornwall, England. In: Environment and Planning A 28, 10, 1847-1867.

Thrift, N. (1977): An introduction to time-geography. Norwich. = Concepts and Techniques in Modern Geography, 13.

Van der Spek, S.; van Schaick, J.; de Bois, P.; de Haan, R. (2009): Sensing Human Activity: GPS Tracking. Sensors 9, 4, 3033-3055.

Weber, H.-J. (2012a): Städtetourismus in Berlin - Möglichkeiten zur GPS-basierten Verortung touristischer Praktiken. In: Zehrer, A.; Grabmüller, A. (Hrsg.): Tourismus $2020+$ interdisziplinär Herausforderungen für Wirtschaft, Umwelt und Gesellschaft. Berlin, 65-78.

Weber, H.-J. (2012b): Die Paradoxie des Städtetourismus - zwischen Massentourismus und Individualität: Eine Studie zu touristischen Praktiken und Mobilität unter Verwendung von GPS- und Fragebogendaten sowie Reiseführerliteratur am Beispiel der Stadt Berlin. Berlin.

Weber, H.-J.; Bauder, M. (2013): Neue Methoden der Mobilitätsanalyse: Die Verbindung von GPS-Tracking mit quantitativen und qualitativen Methoden im Kontext des Tourismus. In: Raumforschung und Raumordnung 71, 2, 99-113.

Zängler, T. (2000): Mikroanalyse des Mobilitätsverhaltens in Alltag und Freizeit. Berlin. 